\title{
Optogenetic actuator/biosensor circuits for large-scale interrogation of ERK dynamics identify sources of MAPK signaling robustness.
}

Coralie Dessauges ${ }^{1}$, Jan Mikelson², Maciej Dobrzyński ${ }^{1}$, Marc-Antoine Jacques ${ }^{1}$, Agne Frismantiene ${ }^{1}$, Paolo Armando Gagliardi ${ }^{1}$, Mustafa Khammash², Olivier Pertz ${ }^{1}$

${ }^{1}$ Institute of Cell Biology, University of Bern, Baltzerstrasse 4, 3012 Bern, Switzerland ${ }^{2}$ Department of Biosystems Science and Engineering, ETH Zurich, Mattenstrasse 26, 4058 Basel, Switzerland

\section{Abstract}

Measurements of single-cell ERK activity dynamics provide unique insights in the MAPK network topology. We built genetic circuits consisting of optogenetic actuators activating ERK from different nodes within the MAPK network together with an ERK biosensor to measure single-cell ERK dynamics. Evaluating ERK dynamics induced by different temporal optogenetic inputs, in response to a large number of perturbations, shows that the MAPK network is robust to downregulation of most of its nodes. This robustness emerges in part because of the ERK-RSK2-SOS negative feedback. Bypassing this feedback, by direct activation of the RAS/RAF/MEK/ERK submodule, or by RSK2 perturbation, breaks MAPK network robustness. Targeting the RSK2-mediated feedback in a ErbB2-dependent oncogenic signaling model greatly sensitizes ERK to MEK inhibition, allowing efficient ERK activity shutdown within a cell population. Thus, the RSK2-mediated negative feedback is a weak node of the MAPK network whose perturbation enables potent inhibition of ERK. 


\section{Introduction}

The extracellular signal-regulated kinase (ERK) is part of the mitogen-activated protein kinase (MAPK) signaling network and regulates a large variety of fate decisions. While ERK can be activated by several extracellular inputs, ERK signaling has mostly been studied in the context of receptor tyrosine kinases (RTKs). Upon binding of their cognate growth factors (GFs), RTKs activate a complex signaling cascade with the following hierarchy: (1) recruitment of adaptor molecules such as GRB2 (Schlessinger, 2000), (2) activation of RAS GTPases through Guanine nucleotide exchange factors (GEFs) and GTPase activating proteins (GAPs) (Cherfils and Zeghouf, 2013), (3) triggering of a tripartite RAF, MEK, ERK kinase cascade that is further regulated by a variety of binding proteins (Lavoie et al., 2020), (4) ERK-mediated phosphorylation of a large number of substrates. Due to its central role in fate decisions, MAPK network dysregulation is causative for a large number of diseases including cancer (Rauen, 2013; Samatar and Poulikakos, 2014).

As for other signaling pathways (Purvis and Lahav, 2013), ERK activity dynamics rather than steady states control fate decisions (Albeck et al., 2013; Avraham and Yarden, 2011; Ryu et al., 2015; Santos et al., 2007). Specific ERK activity dynamics, hereafter referred to as ERK dynamics, have been shown to arise from feedbacks in the MAPK network. For example, negative feedback from ERK to RAF produces adaptive or oscillatory ERK dynamics (Avraham and Yarden, 2011; Kholodenko et al., 2010; Santos et al., 2007). Negative feedbacks also provide network robustness, allowing for high fidelity signal transmission in response to noisy environmental inputs and noise in MAPK network node expression level (Blüthgen and Legewie, 2013; Fritsche-Guenther et al., 2011; Sturm et al., 2010). While several negative feedback loops have been mapped experimentally in the MAPK network (Lake et al., 2016), their role in shaping ERK dynamics remains largely unknown.

Single-cell biosensor imaging has provided new insights into MAPK signaling that were not accessible with biochemical, population-averaged measurements. This showed that the MAPK network can produce a wide variety of ERK dynamics such as transient (Ryu et al., 2015), pulsatile (Albeck et al., 2013), oscillatory (Shankaran et al., 2009) and sustained dynamics (Blum et al., 2019; Ryu et al., 2015), which partly explains how a single pathway can control different fates. Mathematical modeling has provided insight into the network structures that allow decoding of different inputs into specific ERK dynamics (Nakakuki et al., 2010; Ryu et al., 2015; Santos et al., 2007; Shankaran et al., 2009). Combined modeling/experimental approaches captured emergent properties of MAPK subnetworks, including the epidermal growth factor receptor (EGFR) module (Koseska and Bastiaens, 2020), the RAS module (Erickson et al., 2019; Schmick et al., 2015), and the tripartite RAF/MEK/ERK cascade (Arkun and Yasemi, 2018; Ferrell and Bhatt, 1997; Kholodenko, 2000; Kochańczyk et al., 2017; Orton et al., 2005; Ryu et al., 2015; Santos et al., 2007). However, the low experimental throughput to measure ERK dynamics, or other MAPK network nodes, has precluded a global understanding of the specific functions of the nodes present in 
the network. Here, we built genetic circuits consisting of optogenetic actuators to activate ERK from different nodes in the MAPK network, together with an ERK biosensor to measure single-cell ERK dynamics. This allowed us to challenge cells with highly defined temporal inputs and to explore ERK dynamics that result from different MAPK subnetworks. RNA interference (RNAi) screening of 50 signaling nodes revealed that the MAPK network is robust against perturbations of many of its nodes. We found that this emerges, in part, because of the previously documented ERK/RSK2/SOS negative feedback. We show that perturbation of this feedback can weaken the MAPK network in an oncogenic signaling model, allowing to sensitize ERK activation to MEK inhibition, and to robustly shut down ERK activity in all the cells of a population. Thus, identification of the MAPK feedback circuitry provides a rationale to weaken the MAPK network for potent pharmacological inhibition of ERK in cancer. 


\section{Results}

\section{An optogenetic actuator-biosensor genetic circuit to study input-dependent ERK dynamics}

In order to measure ERK dynamics in response to dynamic RTK inputs, we built a genetically-encoded circuit made of an optogenetic RTK actuator and an ERK biosensor (Figure 1A). We chose optoFGFR, which consists of a myristoylated intracellular domain of the fibroblast growth factor receptor 1 (FGFR1) fused to a CRY2 domain (Kim et al., 2014). Upon stimulation with blue light, optoFGFR dimerizes and trans-autophosphorylates, leading to the activation of the MAPK/ERK, phosphoinositide 3-kinase (PI3K)/AKT, and phospholipase $\mathrm{C}(\mathrm{PLC}) / \mathrm{Ca}^{2+}$ pathways. We used ERK-KTR-mRuby2 as an ERK biosensor that is spectrally orthogonal to optoFGFR. ERK-KTR reversibly translocates from the nucleus to the cytosol upon ERK activation (Regot et al., 2014). We used a nuclear Histone 2 B (H2B)-miRFP703 marker to identify and track single cells. These constructs were stably inserted into murine NIH3T3 fibroblasts. We then used automated time-lapse microscopy to stimulate selected fields of view with defined blue light input patterns to activate optoFGFR and record the corresponding ERK-KTR/H2B signals with a 1-minute temporal resolution. We observed that a $100 \mathrm{~ms}$ light pulse leads to reversible ERKKTR translocation from the nucleus to the cytosol, indicative of transient ERK activation (Figure 1B, Movie S1). We built a computer vision pipeline to automatically track each nucleus, and compute ERK activity as the cytosolic/nuclear ratio of the ERK-KTR signals (Figure 1C). At the end of an experiment, we quantified optoFGFR expression levels using a fused mCitrine fluorophore. This allows us to correlate single-cell ERK responses to optoFGFR expression levels. We evaluated the sensitivity and specificity of our system with dose response experiments using the FGFR inhibitor SU5402, the RAF inhibitor RAF709, the MEK inhibitor U0126 and the ERK inhibitor SCH772984 (Figure S1A). The quantification of optoFGFR dimerization kinetics revealed a dissociation time of 5 minutes, assumed to reflect its signaling duration (see Text S1, Figure S1B,C, Movie S2). A short ERK inactivation period manifesting as a dip in the ERK response was observed directly after the light input. We have shown that this dip results from the dephosphorylation of ERK-KTR by Calcineurin, a phosphatase activated by optoFGFR-dependent $\mathrm{Ca}^{2+}$ signaling (Text S1, Figure S1D-F). 
A

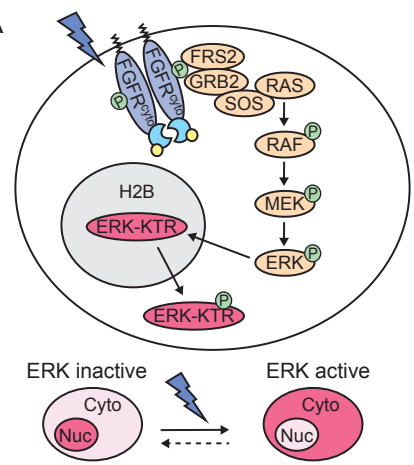

B

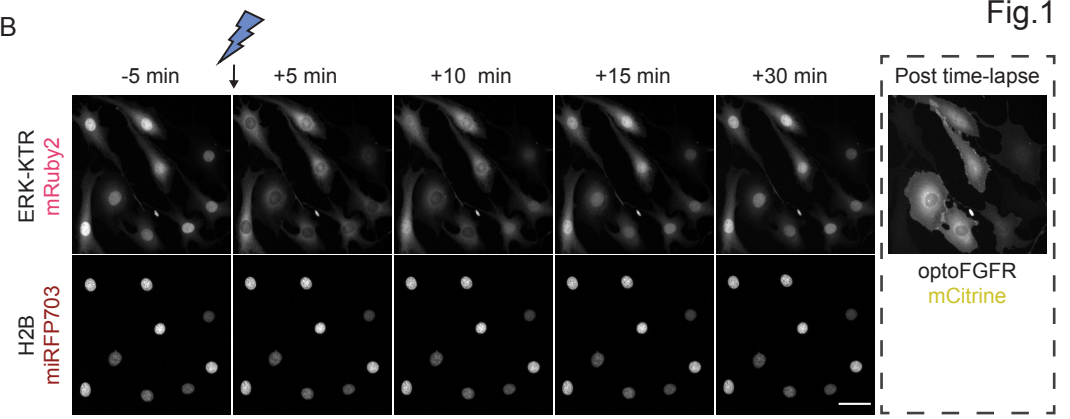

C
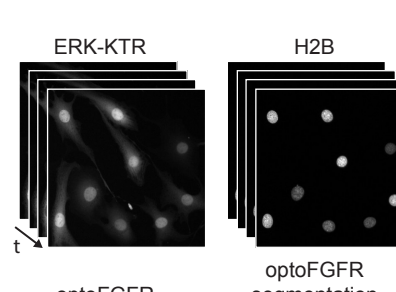

optoFGFR

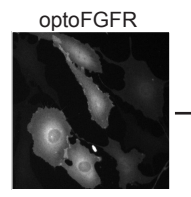
segmentation
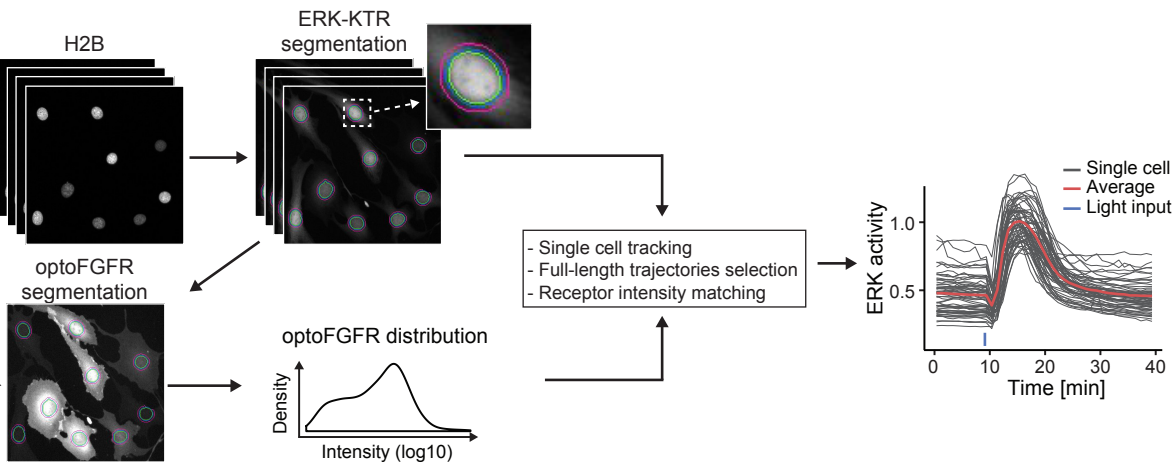

Figure 1: An optogenetic actuator-biosensor genetic circuit to study input-dependent ERK dynamics. (A) Schematic representation of the optoFGFR system consisting of the optogenetic FGF receptor (optoFGFR) tagged with mCitrine, the ERK biosensor (ERK-KTR) tagged with mRuby2 and a nuclear marker (H2B) tagged with miRFP703. (B) Time lapse micrographs of ERK-KTR dynamics in response to a $470 \mathrm{~nm}$ transient light pulse. ERK-KTR and H2B channels were acquired every 1 minute and the optoFGFR channel at the end of the experiment using a 20x air objective. Scale bar: $50 \mu \mathrm{m}$. (C) Image analysis pipeline developed to quantify single-cell ERK responses. Nuclear and cytosolic ERK-KTR signals were segmented based on the H2B nuclear mask. Single-cell ERK activity was then calculated as the cytosolic/nuclear ERK-KTR ratio. Single-cell optoFGFR intensity was measured under the cytosolic ERK-KTR mask and used as a proxy for optoFGFR expression level.

\section{Transient, oscillatory and sustained ERK activity are triggered by different optoFGFR inputs}

Next, we characterized optoFGFR-triggered ERK dynamics in response to a single light pulse of different intensities and durations (Figure 2A). As ERK dynamics depended on light power density and pulse duration, we defined the light dose (D, $\mathrm{mJ} / \mathrm{cm}^{2}$ ) as the product of both to quantify the total energy received per illuminated area. To characterize ERK dynamics, we extracted the amplitude at the maximum of the peak (maxPeak), and the full width at half maximum (FWHM) of the ERK trajectories (Figure 2B). With increasing light doses, ERK peaks increased both in duration and amplitude, until the latter reached saturation. Based on these observations, we selected $180 \mathrm{~mW} / \mathrm{cm}^{2}$ and $100 \mathrm{~ms}\left(D=18 \mathrm{~mJ} / \mathrm{cm}^{2}\right)$ as the minimal light input to generate an ERK transient of maximal amplitude. Using this light dose, we then investigated ERK dynamics in response to multiple light pulses delivered at different intervals (Figure 2C). Repeated light inputs applied at 10- or 20-minutes intervals evoked population-synchronous ERK transients. In contrast, repeated light 
inputs applied at higher frequencies (2-minutes intervals) led to sustained ERK dynamics. Given CRY2's 5-minutes dissociation half-life (Figure S1B,C) (Duan et al., 2017), this suggests that light pulses delivered at a 2-minutes interval reactivate optoFGFR faster than it deactivates, leading to sustained optoFGFR activity. Hierarchical clustering of ERK responses to sustained optoFGFR inputs revealed sustained and oscillatory single-cell ERK dynamics (Figure 2D). Classification of ERK trajectories based on optoFGFR expression revealed that sustained/oscillatory ERK dynamics correlated with high/low optoFGFR levels (Figure 2E, Movie S3). Oscillatory ERK dynamics were also observed in optoFGFR high expressing cells in response to low light input (Figure 2F). Thus, sustained and oscillatory ERK dynamics can be triggered in response to different optoFGFR input strengths - a combination of light energy and optoFGFR expression.

To further understand optoFGFR-evoked ERK dynamics, we compared it to sustained FGFR and EGFR inputs. Stimulation with basic FGF led to sustained ERK dynamics that exhibited a biphasic behavior in a dose response challenge (Figure S2A,B). This was previously shown to emerge from competition of basic FGF for FGFR and heparan sulfate proteoglycan co-receptors (Blum et al., 2019). The ability of optoFGFR to trigger different ERK dynamics thus can be explained because of a lack of these extracellular interactions. EGF stimulation, on the other hand, induced an ERK transient with prominent adaptation (Figure S2C,D), which has been shown to involve negative feedback (Avraham and Yarden, 2011; Kholodenko et al., 2010; Santos et al., 2007). However, similarly to low optoFGFR input, low FGFR and EGFR inputs induced oscillatory ERK behavior. To understand the origin of the oscillatory ERK dynamics, as well as the difference in ERK dynamics evoked by EGFR and optoFGFR inputs, we sequentially applied EGFR and optoFGFR inputs to the same cells (Figure S2E) and observed loss of ERK oscillations upon low optoFGFR input. Mathematical modeling of these experiments revealed that ERK oscillations emerge from a conserved negative feedback downstream of optoFGFR and EGFR, and that optoFGFR lacks receptor-level regulatory mechanisms relevant to the endogenous EGFR (Figure S3). These experiments and additional modeling efforts are discussed thoroughly in Text S2. While optoFGFR lacks some regulatory mechanisms relevant to endogenous RTKs, it faithfully captures several features of RTK/MAPK signaling. This includes the steep increase in ERK activity when the input is applied, the characteristic adaptation kinetics observed upon optoFGFR input removal, as well as the ability to induce oscillatory ERK dynamics in response to low input. 
A

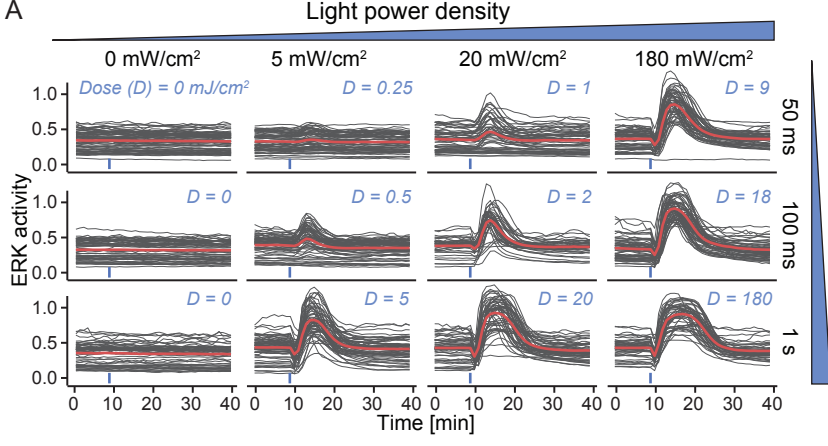

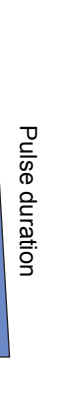

Feature extraction

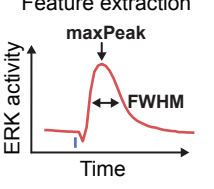

Power density

$00 \mathrm{~mW} / \mathrm{cm}^{2}$

$0 \mathrm{~mW} / \mathrm{cm}^{2}$
$0 \mathrm{~mW} / \mathrm{cm}^{2}$

प $20 \mathrm{~mW} / \mathrm{cm}^{2}$

$180 \mathrm{~mW} / \mathrm{cm}^{2}$

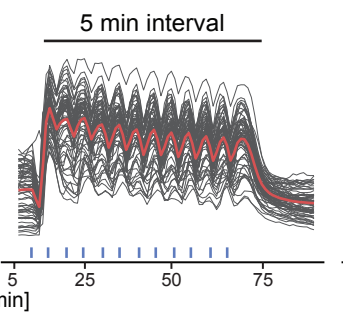

E
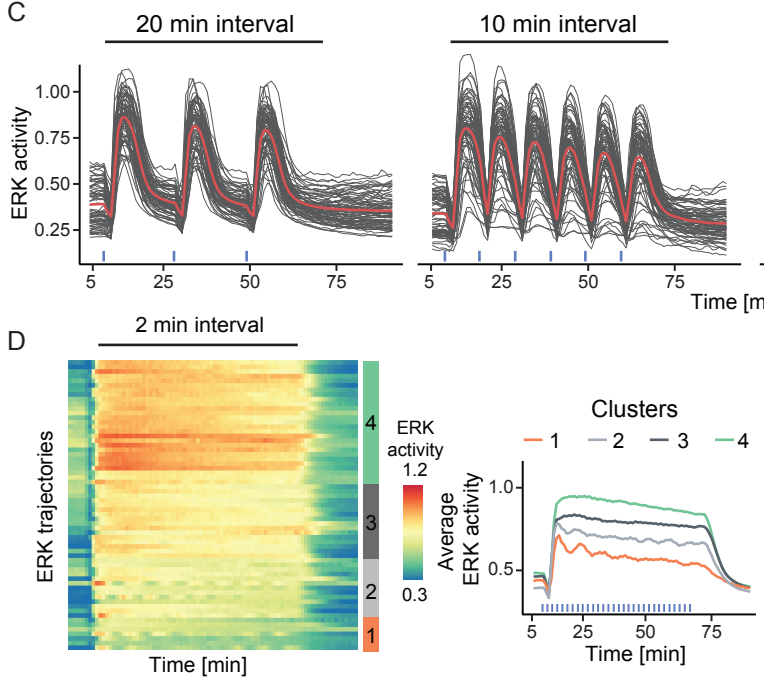

C
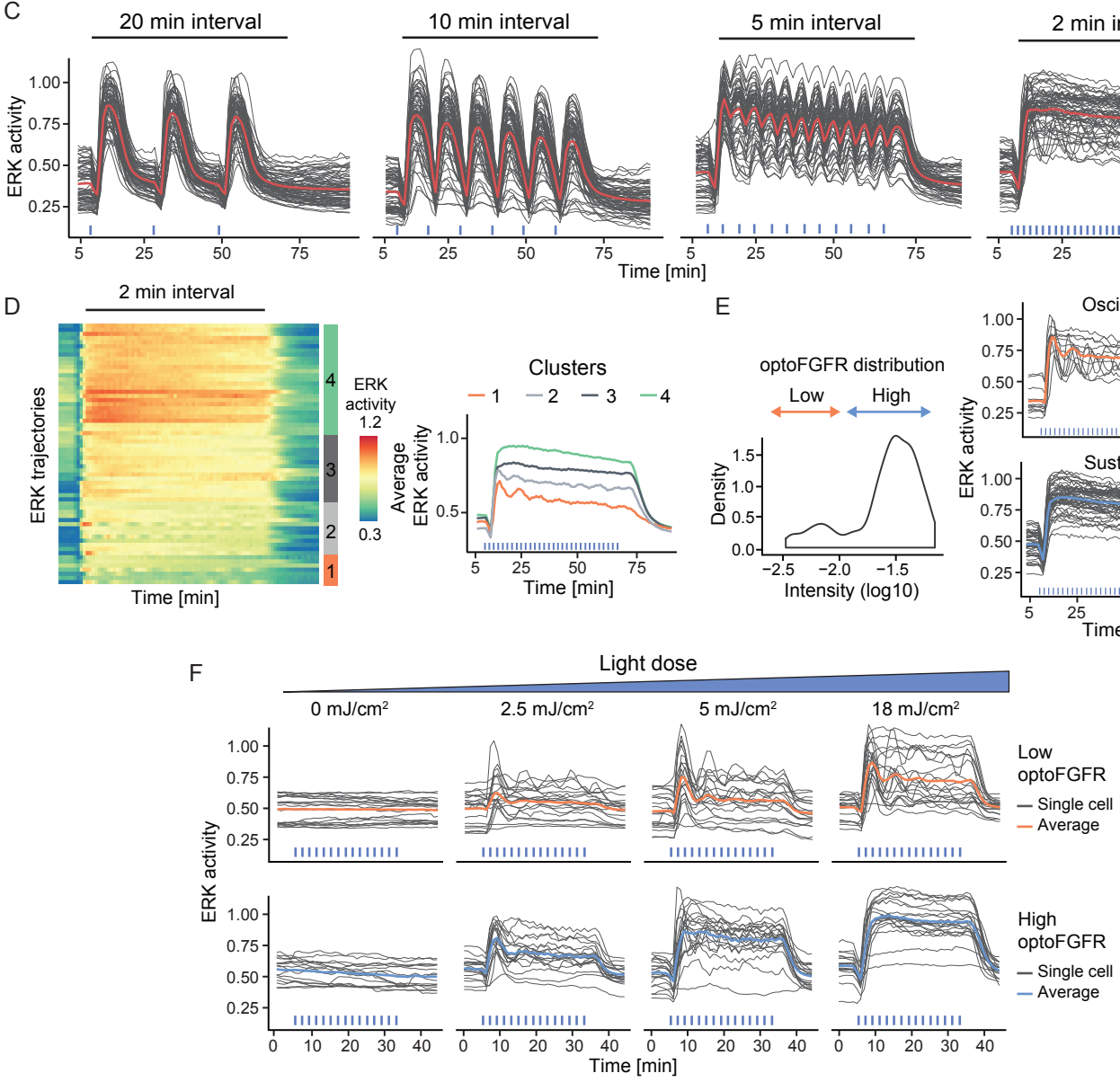

Figure 2: Transient, oscillatory and sustained ERK activity are triggered by different optoFGFR inputs. (A) ERK responses to increasing light power densities and pulse durations of a $470 \mathrm{~nm}$ transient light input. The light dose " $D$ " is calculated as the product of the power density and pulse duration. (B) Quantification of the MaxPeak (maximal ERK amplitude of the trajectory) and the FWHM (full width at half maximum, the peak duration at half maxPeak) of single-cell ERK responses shown in (A) $\left(\mathrm{N}_{\min }=\right.$ 40 cells per condition). (C) Cells were stimulated with $470 \mathrm{~nm}$ light pulses delivered every 20, 10, 5 and 2 minutes respectively $\left(D=18 \mathrm{~mJ} / \mathrm{cm}^{2}\right)$. (D) Hierarchical clustering (Euclidean distance and Ward D2 linkage) of trajectories from the 2-minute interval (referred to as "sustained") stimulation shown in (C) $(\mathrm{N}=60$ cells). The number of clusters was empirically defined to resolve different ERK dynamics. The average ERK response per cluster is displayed on the right. (E) Separation of the trajectories shown in (D) in low and high optoFGFR cells, based on the log10 intensity of optoFGFR-mCitrine. (F) ERK responses to increasing doses of sustained optoFGFR inputs. Single-cell ERK trajectories were divided in low (top panel) and high (bottom panel) optoFGFR expression. 


\section{Large-scale perturbation of the MAPK network reveals robustness against downregulation of its signaling nodes}

To explore the network circuitry that shapes ERK dynamics, we performed an RNA interference (RNAi) screen and probed the effect of node perturbations in response to sustained optoFGFR input. We used a bioinformatic approach to identify a potential MAPK network by sequentially querying known interactors of the tripartite RAF/MEK/ERK cascade downstream of the FGFR receptor that were detected in a NIH3T3 proteome (Schwanhäusser et al., 2011) (Figure 3A, Table S4). This network consisted of a receptor proximal layer containing adaptors (GRB2, FRS2), negative regulators such as Sprouty proteins and protein tyrosine phosphatases, a RAScentered layer consisting of GEFs, GAPs, and RAS isoforms, a MAPK-centered layer containing MAPK isoforms and scaffold proteins, and a layer of dual specificity phosphatases (DUSPs), documented to deactivate different MAPKs (Patterson et al., 2009). We used the siPOOL technology to specifically knockdown (KD) these 50 MAPK signaling nodes while limiting off-target effects (Hannus et al., 2014). We validated $K D$ efficiency by quantifying transcript levels with different siPOOL concentrations targeting ERK and MEK isoforms (Figure S4A) and observed strong $\mathrm{KD}$ with $10 \mathrm{nM}$ siRNA concentration. We then evaluated the effect of ERK1 and ERK2 KD on ERK dynamics. Surprisingly, we observed only subtle phenotypes (Figure 3B), even though efficient KD was observed at protein level (Figure 3C). However, combined ERK1/ERK2 KD suppressed ERK dynamics showing that ERK activation is robust to perturbation of individual ERK isoforms as previously reported (FritscheGuenther et al., 2011; Ornitz and Itoh, 2015). ERK1/ERK2 KD was used as positive control, and a non-targeting siRNA was used as negative control throughout our screens. We performed three replicates of the screen.

We first quantified maximal ERK amplitudes (maxPeak) of cells expressing high optoFGFR levels to exclude noisy oscillatory ERK trajectories (Figure 3D). Many KDs led to statistically significant, albeit mild, ERK amplitude phenotypes, despite selective and efficient KD quantified for several nodes (Figure S4B). Strongly decreased ERK amplitudes were observed for GRB2, ERK2 and RSK2 KD, and an increase in amplitudes was observed for KD of a panel of DUSPs, and some negative regulators such as PP2A and Sproutys (Figure 3D).

We then investigated changes in the shape and heterogeneity of ERK trajectories. Visual evaluation of population-averaged ERK dynamics revealed some penetrant phenotypes (Figure S4C,D). However, the high heterogeneity in single-cell trajectories precluded the definition of a minimal set of human-defined features to capture all possible ERK dynamics phenotypes in an unbiased fashion. To bypass this limitation, we used CODEX (Jacques et al., 2021), a data-driven approach to identify patterns in single-cell time-series based on convolutional neural networks (CNNs) (Figure S5A). We trained a CNN to classify ERK trajectories that originate from different siRNA perturbations, irrespectively of optoFGFR expression levels. To identify perturbations that induced distinct ERK dynamics, we selected the ten perturbations for which the $\mathrm{CNN}$ classification accuracy was the highest (Table S4, "CODEX accuracy"). We then 
trained a second model on those ten perturbations. Projection of the CNN features in a t-distributed stochastic neighbor embedding (t-SNE) space revealed well-separated clusters of ERK trajectories (Figure S5B). We then displayed the ten trajectories with the highest classification confidence for each perturbation to get intuition about the effect of the perturbation on ERK trajectories (Figure 3E, "CODEX"). Comparison with randomly selected ERK trajectories for both optoFGFR-low (oscillatory ERK dynamics) and optoFGFR-high cells (sustained ERK dynamics) confirmed these phenotypes (Figure 3E). As expected, ERK1+ERK2 KD completely inhibited ERK activity. KD of the general phosphatase $P P 2 A$ induced oscillatory behavior with high amplitude. RSK2 KD led to strongly decreased oscillatory ERK behavior, slower deactivation kinetics upon input removal (Figure S5C), and increased heterogeneity in ERK amplitude decreasing population-averaged ERK amplitude. PLCG1 KD resulted in shorter ERK peaks, increased oscillatory behavior, as well as absence of the dip. As phospholipase $\mathrm{C}$ mediates $\mathrm{Ca}^{2+}$ signaling in response to FGFR activation (Ornitz and Itoh, 2015), this further validates the role of $\mathrm{Ca}^{2+}$ signaling in formation of the dip (Figure S1D-F). ERK2 KD led to a slower ERK activation rate, decreased oscillatory behavior and lower overall ERK amplitude. DUSP6 KD led to increased ERK amplitude and baseline ERK activity levels (Figure S5C). GRB2 KD, and to a lower extent FRS2 KD, led to heterogeneous ERK dynamics with a large cell population not being able to produce robust ERK responses, decreasing population-averaged ERK amplitude. RAPGEF1 KD led to increased oscillatory behavior in optoFGFR-low cells. CRAF KD led to strongly decreased oscillatory behavior, and increased heterogeneity in ERK amplitudes. While other perturbations led to subtle changes in ERK amplitudes, they did not induce detectable ERK dynamics phenotypes, suggesting that the MAPK network is robust against RNAi perturbation of most of its nodes under sustained input.

As our modeling efforts suggest (Figure S3, Text S2), oscillatory ERK behavior might emerge from the presence of negative feedback in the MAPK network (Kholodenko, 2000). Therefore, perturbations decreasing ERK oscillations might identify nodes involved in such feedback regulation. We systematically evaluated the proportion of oscillatory versus non-oscillatory ERK trajectories in response to each perturbation, for both high and low optoFGFR levels (Figure S5D). This confirmed increased ERK oscillations for PP2A and PLCG1 KD and decreased ERK oscillations for RSK2, CRAF and ERK2 KD. To validate these findings in the context of an endogenous RTK, we evaluated ERK2, CRAF and RSK2 KD in cells stimulated with $1 \mathrm{ng} / \mathrm{ml} E G F$, which leads to prominent ERK oscillations (Figure S2C). ERK2 KD decreased ERK activation rate and amplitude, while CRAF and RSK2 KD decreased ERK oscillations (Figure S5E), which was confirmed with hierarchical clustering (Figure S5F,G). The ERK2 and CRAF isoforms might therefore be specifically involved in the negative feedback in which ERK phosphorylates and negatively regulates RAF activity (Fritsche-Guenther et al., 2011). RSK2 encodes the p90 ribosomal S6 kinase 2, an ERK substrate that mediates negative feedback at the level of the GEF SOS (Douville and Downward, 1997; Lake et al., 2016; Saha et al., 2012). This suggests that two negative feedbacks, one from ERK to RAF, and the other from ERK to SOS via RSK2, simultaneously 
bioRxiv preprint doi: https://doi org/10.1101/2021.07 27.453955; this version posted July 27, 2021. The copyright holder for this preprint (which was not certified by peer review) is the author/funder, who has granted bioRxiv a license to display the preprint in perpetuity. It is made available under aCC-BY-NC-ND 4.0 International license.

operate within the MAPK network in order to generate ERK oscillations (Figure 4A). As negative feedback enables network robustness against noise in signaling node expression levels (Fritsche-Guenther et al., 2011), two negative feedbacks acting at different levels in the MAPK network might be advantageous to warrant this robustness.
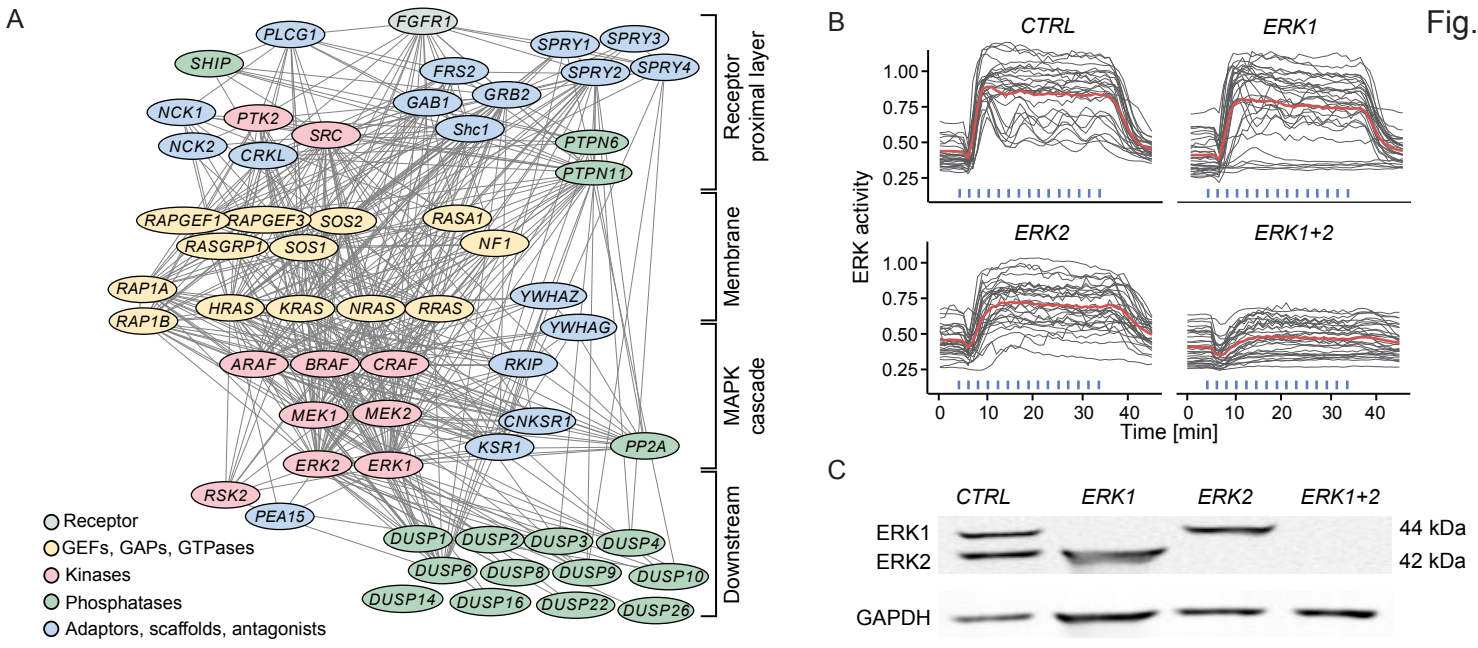

$\mathrm{D}$

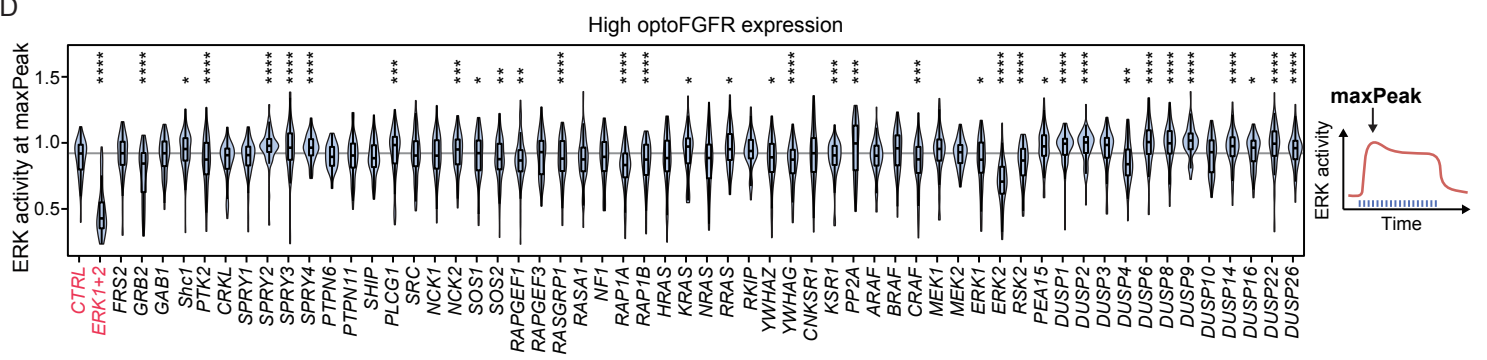

$\mathrm{E}$
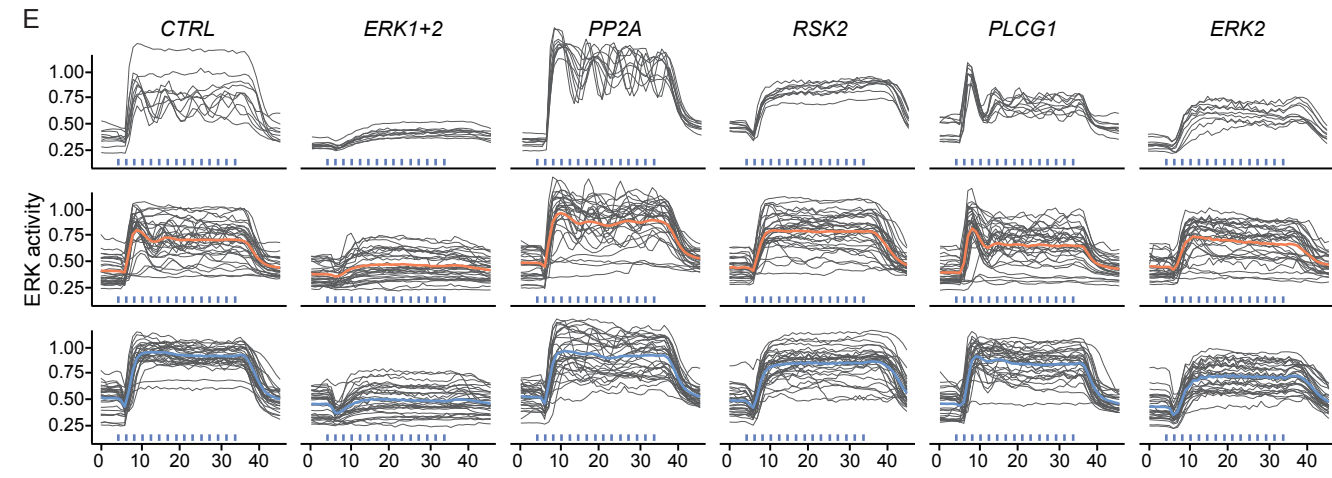

CODEX prototype
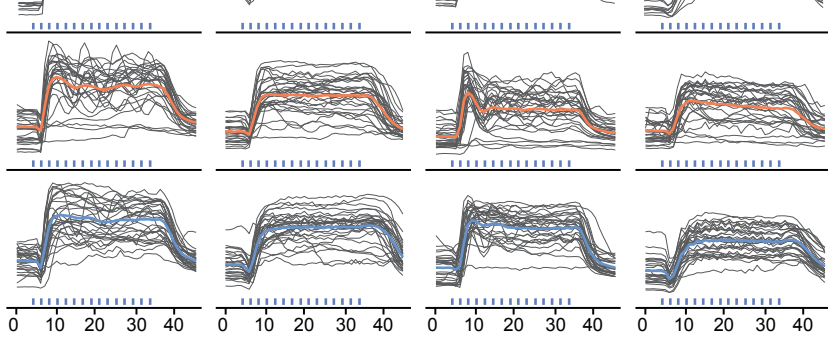
Low andom
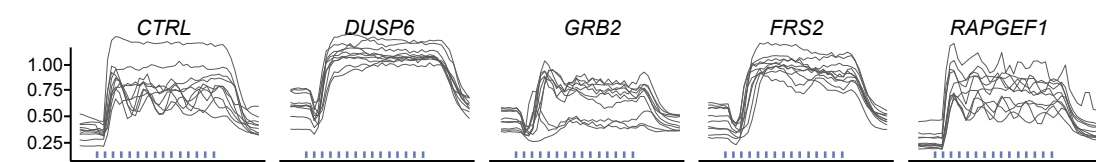

CRAF
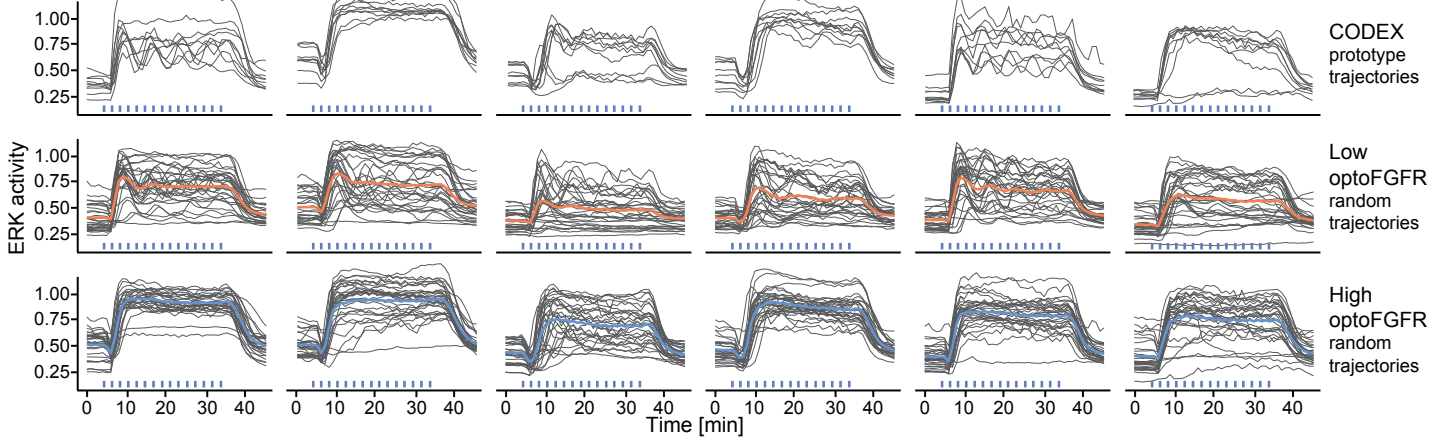
Figure 3: Large-scale perturbation of the MAPK network reveals robustness against downregulation of its signaling nodes. (A) RNAi perturbation targets. Signaling nodes were spatially grouped based on the hierarchy of interactions within the MAPK network and color-coded for their function. (B) ERK responses to sustained optoFGFR inputs $\left(D=18 \mathrm{~mJ} / \mathrm{cm}^{2}\right)$ in cells transfected with $10 \mathrm{nM}$ siRNA against ERK1, ERK2 or a $5 \mathrm{nM}$ combination of each (ERK1+2). A non-targeting siRNA (CTRL) was used as control ( $\mathrm{N}=15$ cells from low and high optoFGFR levels). (C) Western blot analysis of cells transfected with $10 \mathrm{nM}$ siRNA against ERK1, ERK2 or a $5 \mathrm{nM}$ combination of each $(E R K 1+2)$. (D) Quantification of the maxPeak of single-cell ERK responses under sustained high optoFGFR inputs $\left(\mathrm{D}=18 \mathrm{~mJ} / \mathrm{cm}^{2}\right)$ for each RNAi perturbation ( $\mathrm{N}=60$ cells per treatment, from 3 technical replicates). Statistical analysis was done using a Wilcoxon test comparing each perturbation against the nontargeting siRNA (CTRL) $\left(\mathrm{N}_{\min }=120\right.$ cells per condition, ${ }^{*}<0.05,{ }^{* *}<0.005,{ }^{* * *}<0.0005,{ }^{* * * *}<0.00005$, FDR p-value correction method). (E) Single-cell ERK trajectories in response to sustained optoFGFR inputs $\left(D=18 \mathrm{~mJ} / \mathrm{cm}^{2}\right)$ for the RNAi perturbations classified with the highest accuracy by CODEX. Top line shows single-cell ERK trajectories for which CODEX had the highest classification confidence in the validation set $(\mathrm{N}=10)$. Bottom lines show single-cell ERK trajectories for low and high optoFGFR cells $(\mathrm{N}=30$ for each, randomly selected out of at least 212 cells per perturbation from 3 technical replicates). For better visualization, the CTRL condition is shown twice.

\section{The RAS/ERK subnetwork is less robust against node perturbation than the whole optoFGFR-triggered MAPK network}

To explore the significance of the two feedbacks in the MAPK network, we used optoSOS, an optogenetic actuator that activates RAS (Johnson et al., 2017). OptoSOS directly induces the RAS/RAF/MEK/ERK subnetwork (referred to as the RAS/ERK subnetwork), bypassing any feedback regulation upstream of RAS, such as the ERK/RSK2/SOS negative feedback (Douville and Downward, 1997; Lake et al., 2016; Saha et al., 2012) (Figure 4A). OptoSOS consists of a membrane anchored lightactivatable iLID domain, and an mCitrine-tagged SspB domain fused to SOS's catalytic GEF domain. It was stably integrated into cells expressing ERK-KTR and H2B. Because iLID displays faster dissociation rates than CRY2 $\left(\mathrm{t}_{1 / 2}=30\right.$ seconds for iLID versus $\sim 5$ minutes for CRY2 (Benedetti et al., 2018; Duan et al., 2017)), optoSOS required repeated light pulses for prolonged membrane recruitment to produce a robust ERK response. A single light pulse resulted in a low amplitude ERK transient, even at high light dose (Figure 4B, "1x"). Five consecutive $100 \mathrm{~ms}$ light pulses at 6 $\mathrm{W} / \mathrm{cm}^{2}$ light pulses $\left(D=0.6 \mathrm{~J} / \mathrm{cm}^{2}\right)$ applied at 20-second intervals, provided the minimal light input to induce a saturated ERK amplitude (Figure S6A). Application of this light input at 2-minute intervals evoked sustained ERK dynamics with small fluctuations at the same frequency than the light input pattern, reflecting the fast optoSOS reversion to the dark state (Figure 4C). OptoSOS did not induce ERK oscillations (Figure S6B), even in optoSOS low cells or for lower light doses (Figure 4D). However, ERK amplitudes correlated with optoSOS expression level - low versus high optoSOS levels leading to low versus high ERK amplitudes. Using the minimal light input to trigger saturating ERK amplitude, both optoSOS and optoFGFR led to steep ERK activation and fast adaptation when light stimulation ceased (compare Figures $2 \mathrm{C}$ and $4 \mathrm{C}$ ), as well as similar ERK amplitudes in cells with high optogenetic actuator levels (Figure 4E). However, optoSOS-evoked ERK dynamics displayed desensitization on timescales of tens of minutes and did not exhibit the ERK dip. 
After establishing the light regime that induces ERK responses comparable to those induced by optoFGFR, we performed an siRNA screen targeting only the network nodes downstream of optoSOS (Figure S6C,D). As optoSOS evoked simpler ERK dynamics than optoFGFR, we evaluated the effect of the perturbation by extracting ERK amplitudes in optoSOS-high cells (Figure 5A, compared to 3D). We compared the penetrance of the perturbations in both optogenetic systems, by standardizing the ERK amplitudes with the control treatment (Figure 5B). Most perturbations affected ERK amplitude in a similar way. However, several perturbations led to a more penetrant phenotype in the optoSOS than in the optoFGFR system. This was true but not restricted to perturbation of RAS and RAP isoforms (RASA1, RAP1A, RAP1B, HRAS, NRAS), YWHAZ, PP2A, MEK1, MEK2, ERK1 and ERK2, and a number of DUSPs (DUSP10, DUSP14, DUSP16, DUSP22, DUSP26). In addition, CRAF KD led to an almost complete loss of ERK activity in the optoSOS compared to the optoFGFR system. Further evaluation of ERK dynamics phenotypes illustrated these findings ERK1, ERK2 and CRAF KD led to lower ERK activity, while PP2A and DUSP22 KD led to a prominent increase in ERK amplitude (compare Figure 5C and $3 \mathrm{E}$ ). Our results therefore suggest that the RAS/ERK subnetwork is less resilient against node perturbations than the full MAPK network.

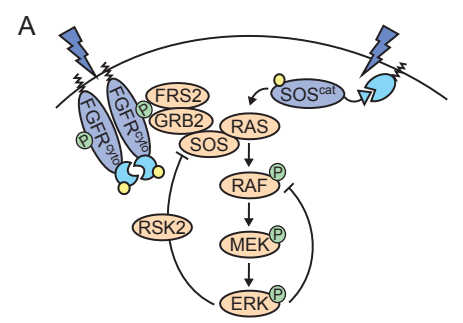

B

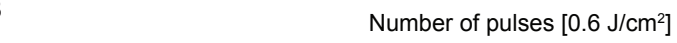

Fig.4
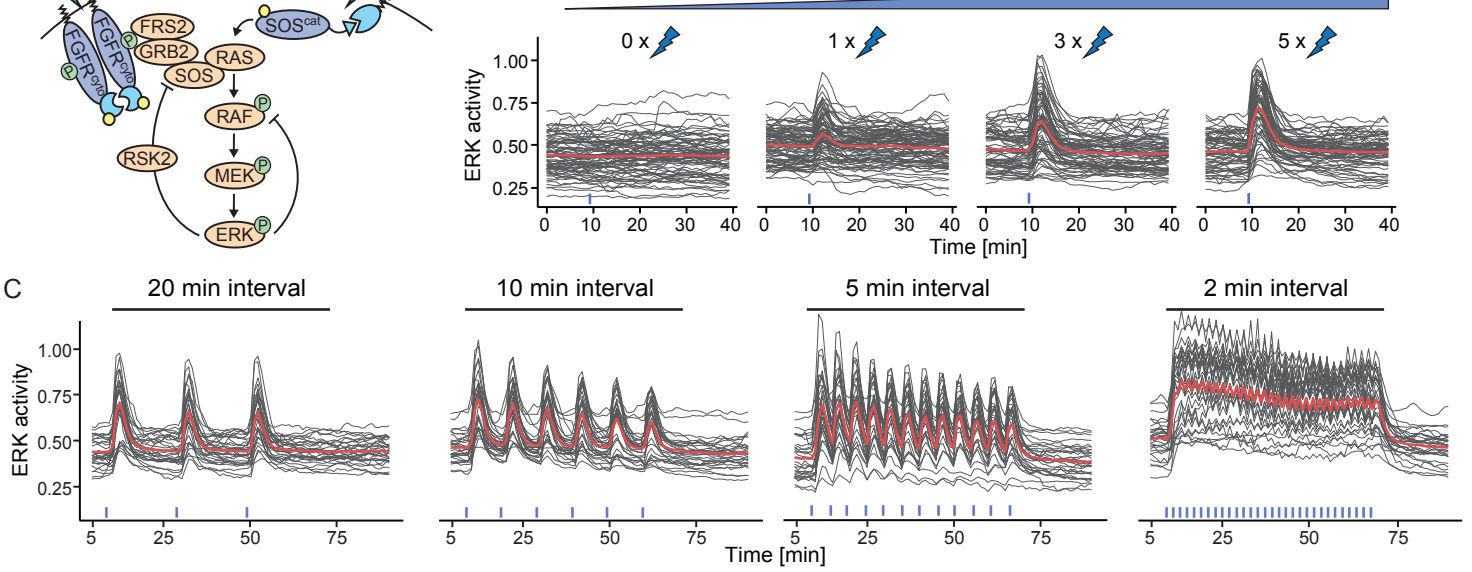

D
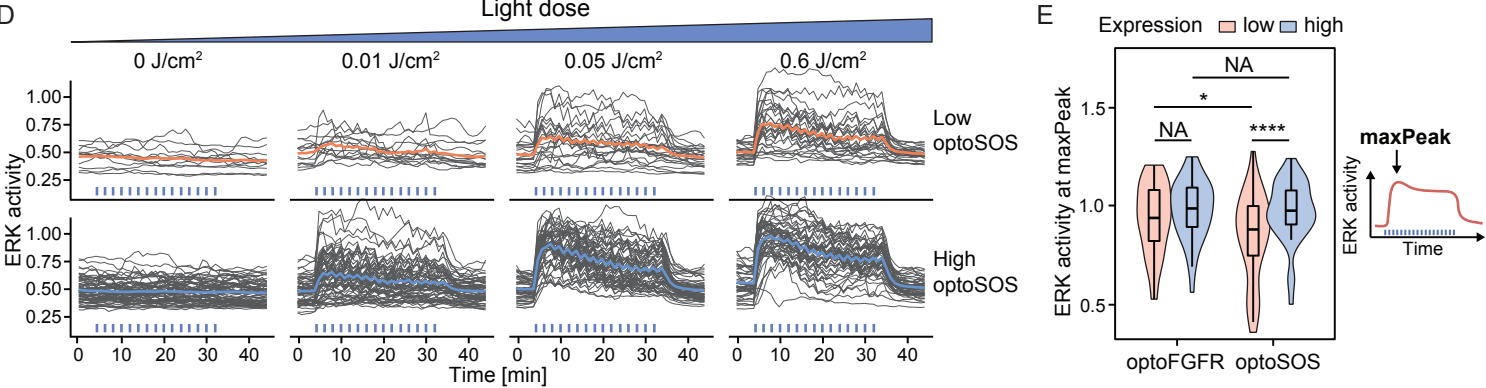

Figure 4: OptoSOS sustained inputs trigger ERK dynamics without oscillatory behavior. (A) Schematic representation of ERK signaling induced by optoSOS versus optoFGFR inputs. (B) ERK dose responses under transient optoSOS inputs consisting of different numbers of repeated $470 \mathrm{~nm}$ pulses $\left(1 \mathrm{x}, 2 \mathrm{x}, 3 \mathrm{x}, 4 \mathrm{x}\right.$ and $5 \mathrm{x}$ pulses applied at 20 -second intervals, $\left.\mathrm{D}=0.6 \mathrm{~J} / \mathrm{cm}^{2}\right)$. Repeated pulses are depicted as a single stimulation bar (blue). (C) Cells expressing optosos were stimulated with 5 repeated $470 \mathrm{~nm}$ light pulses delivered every $20,10,5$ and 2 minutes respectively $\left(D=0.6 \mathrm{~J} / \mathrm{cm}^{2}\right)$. (D) 
ERK responses to increasing light doses of sustained optoSOS inputs consisting of 2-minutes interval inputs, each input made of 5 repeated light pulses. Cells were divided in low and high optoSOS expression levels based on the log10 intensity of the optoSOS-mCitrine. (E) Quantification of the maxPeak of single-cell ERK responses under sustained optoFGFR (Figure $2 F, D=18 \mathrm{~mJ} / \mathrm{cm}^{2}$ ) and optoSOS (Figure 4D, D = $0.6 \mathrm{~J} / \mathrm{cm}^{2}$ ) inputs for low or high expression of each optogenetic system ( $\mathrm{N}=$ 40 cells per condition). Statistical analysis was done using a Wilcoxon test, comparing each perturbation against the CTRL $\left(\mathrm{N}_{\min }=48\right.$ cells per condition, ${ }^{*}<0.05,{ }^{* *}<0.005,{ }^{* * *}<0.0005,{ }^{* * * *}<0.00005$, FDR pvalue correction method).

A

Fig.5

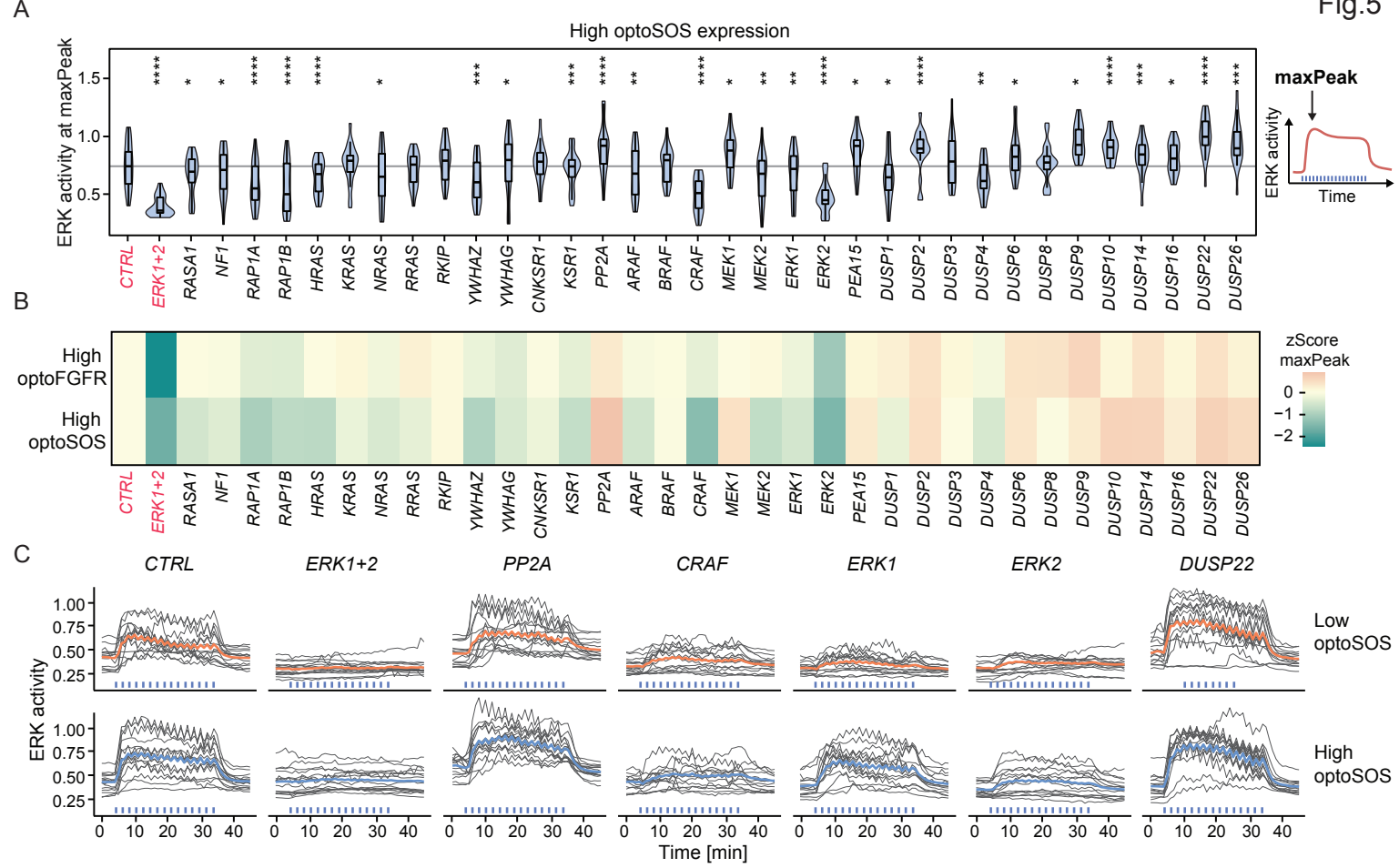

Figure 5: The RAS/ERK subnetwork is less robust against node perturbation than the whole optoFGFR-triggered MAPK network. (A) Quantification of the maxPeak for single-cell ERK responses induced by sustained high optoSOS inputs $\left(D=0.6 \mathrm{~J} / \mathrm{cm}^{2}\right)$ for RNAi perturbations targeting nodes of the RAS and ERK subnetwork ( $N=20$ cells per treatment). Statistical analysis was done using a Wilcoxon test, comparing each perturbation against the CTRL $\left(\mathrm{N}_{\min }=20\right.$ cells per perturbation, ${ }^{*}<0.05$, ${ }^{* *}<0.005,{ }^{* * *}<0.0005,{ }^{* * * *}<0.00005$, FDR p-value correction method). (B) z-Score comparing ERK maxPeak under RNAi perturbations of the RAS/ERK subnetwork to the non-targeting siRNA (CTRL) for high optoSOS and high optoFGFR (Figure 3D) sustained inputs. (C) Single-cell ERK trajectories for low and high optoSOS cells ( $\mathrm{N}=15$ each) for selected RNAi perturbations.

\section{The RSK2 negative feedback confers robustness to the MAPK network against MEK inhibition}

To further compare the robustness of the MAPK network to the RAS/ERK subnetwork, we performed dose response experiments with the MEK inhibitor U0126 in response to sustained optoFGFR and optoSOS inputs (Figure 6A). As some perturbations led to complete loss of ERK activity, we quantified ERK amplitudes at a fixed time point ( $\left.\mathrm{t}_{\text {fixed }}\right)$ that corresponds to the maximum amplitude in the control (Figure 6B). We observed that ERK amplitudes decreased in optoFGFR cells with increasing U0126 doses, but that the population inhibition remained largely heterogeneous for 
concentrations below $20 \mu \mathrm{M}$ (Figure 6B,C). In contrast, optoSOS-evoked ERK dynamics were more sensitive to increasing U1026 and the ERK amplitude heterogeneity decreased at lower doses, leading to lower $\mathrm{IC}_{50}$ values for optoSOS than for optoFGFR-evoked ERK dynamics (Figure 6D). We hypothesized that the sensitivity of the optoSOS-triggered RAS/ERK subnetwork arises from the absence of the RSK2-mediated feedback that operates upstream of SOS. We therefore evaluated optoFGFR-triggered ERK dynamics in response to MEK perturbation in RSK2 KD cells (Figure 6E-H). RSK2 KD decreased ERK heterogeneity at lower U0126 concentrations (Figure $6 E, G$ ), and reduced the $\mathrm{IC}_{50}$ of U0126-mediated ERK inhibition (Figure $6 \mathrm{H}$ ). Similar results were obtained with SL0101, a RSK inhibitor (Smith et al., 2005) (Figure S7A-D). Thus, loss of the RSK2-mediated feedback, either by direct activation of the RAS-ERK subnetwork, or by RSK2 perturbation, decreases the network robustness to MEK perturbation.
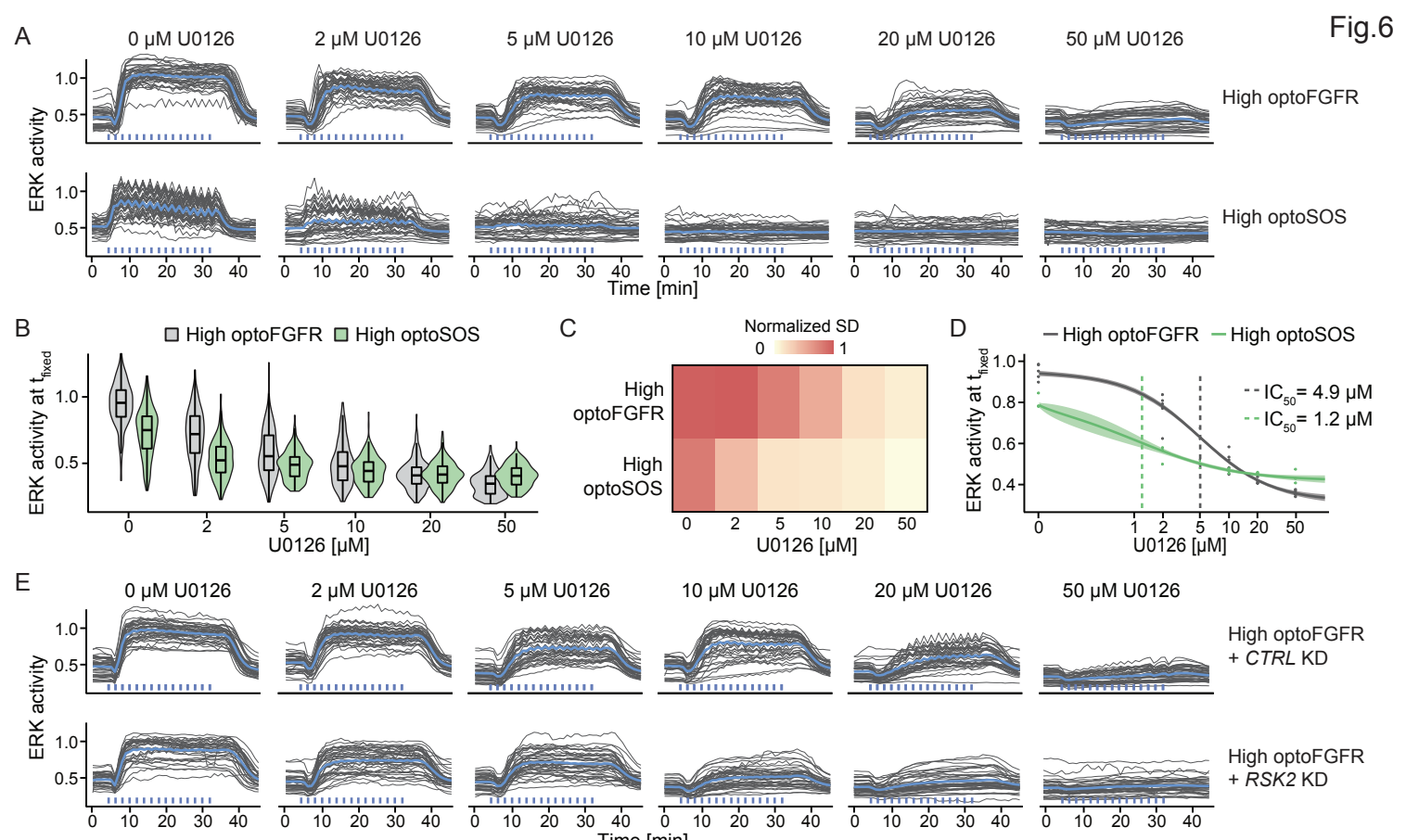

$20 \mu \mathrm{M} \cup 0126$

$50 \mu \mathrm{M} \cup 0126$
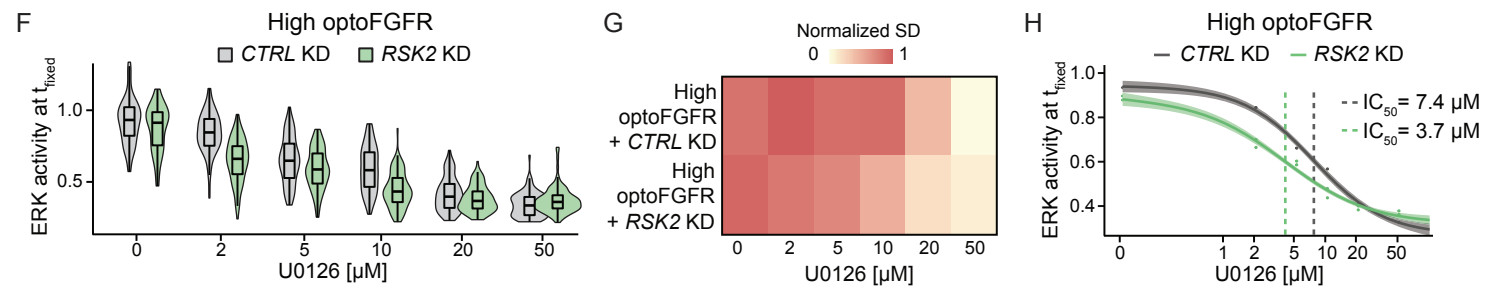

Figure 6: The RSK2 negative feedback confers robustness to the MAPK network against MEK inhibition. (A) Single-cell ERK responses to sustained high optoFGFR $\left(D=18 \mathrm{~mJ} / \mathrm{cm}^{2}\right)$ or optoSOS $\left(D=0.6 \mathrm{~J} / \mathrm{cm}^{2}\right)$ inputs under increasing concentrations of $\mathrm{U} 0126(\mathrm{~N}=40$ cells per condition, randomly selected out of at least 200 cells from 3 technical replicates). (B) ERK responses were extracted at a fixed time point from single-cell trajectories shown in (A) ( $\mathrm{t}_{\text {fixed optoFGFR }}=10$ minutes, $\mathrm{t}_{\text {fixed optosos }}=7$ minutes, $N=200$ cells per condition). (C) Normalized standard deviation of ERK amplitudes shown in (A) $\left(\mathrm{N}_{\min }=200\right.$ cells per perturbation). (D) A Hill function was fit to the mean ERK activity as shown in (A). Shaded area indicates the $95 \% \mathrm{Cl}$ and dashed lines the IC50. (E) Single-cell ERK responses to 
sustained high optoFGFR inputs $\left(D=18 \mathrm{~mJ} / \mathrm{cm}^{2}\right)$ in $C T R L$ or $R S K 2 \mathrm{KD}$ cells under increasing concentrations of $\mathrm{U} 0126$ ( $\mathrm{N}=40$ cells per condition (apart from RSK2 KD + $0 \mu \mathrm{M} \cup 0126$ (32 cells), from 2 technical replicates for $R S K 2 \mathrm{KD}$ and 1 replicate for CTRL KD). (F) ERK responses were extracted at $\mathrm{t}_{\text {fixed }}=10$ minutes from single-cell trajectories shown in (E) $(\mathrm{N}=100$ cells per condition (except for $R S K 2$ $\mathrm{KD}+0 \mu \mathrm{M} \cup 0126)$ ). (G) Normalized standard deviation of ERK amplitudes shown in (E) $\left(\mathrm{N}_{\text {min }}=100\right.$ cells per perturbation (apart from RSK2 KD + $0 \mu \mathrm{M}$ U0126)). (H) A Hill function was fitted to the mean ERK activity as shown in (E). Shaded area indicates the $95 \% \mathrm{Cl}$ and dashed lines the IC50.

\section{Targeting the RSK2-mediated feedback in combination with MEK inhibition potently inhibits population-level ERK dynamics in response to EGFR and oncogenic ErbB2 inputs}

Our results highlighted the role of the RSK2-mediated feedback in the MAPK network robustness against node perturbations in NIH3T3 cells. To test if it also contributes to network robustness in a disease-relevant system, we evaluated its function in nontumorigenic wild-type (WT) MCF10A breast epithelial cells, as well as in ErbB2overexpressing MCF10A cells, that recapitulate ErbB2 amplification observed in $20 \%$ of all breast cancers (Arteaga and Engelman, 2014; Yarden and Pines, 2012). We chose this specific model system because ErbB2 amplification leads to a constitutive RTK input on the MAPK network, with an intact downstream feedback structure, in contrast to mutated RAS or RAF signaling. MCF10A WT cells display asynchronous low frequency ERK pulses in the absence of EGF, and high frequency ERK pulses in the presence of EGF (Figure S7E) (Albeck et al., 2013). On the other hand, MCF10A ErbB2 overexpressing cells displayed high ERK pulse frequencies in the absence of EGF. Previous work has highlighted the role of negative feedbacks in ERK pulse formation (Kochańczyk et al., 2017), suggesting that EGFR and ErbB2 trigger a MAPK network with similar feedback circuitry as in our optoFGFR fibroblast system. Thus, we reasoned that destabilizing network robustness with RSK2 perturbation might potential MEK-mediated ERK inhibition (Figure 7A). We first identified conditions that lead to partial ERK inhibition by performing a U0126 dose response and evaluating ERK amplitudes directly after acute EGF stimulation (Figure S7F). We found that $3 \mu \mathrm{M}$ U0126 decreased the median ERK amplitudes, but still led to considerable ERK amplitude heterogeneity. In population-averaged measurements of EGF-stimulated MCF10A WT cells, $3 \mu \mathrm{M}$ U0126 or $50 \mu \mathrm{M}$ SL0101, led to a partial decrease in ERK activity, while their combination led to potent ERK inhibition (Figure 7B). A similar effect was observed in ErbB2-overexpressing cells (Figure 7C). However, averaging asynchronous ERK dynamics only provides limited insights into single-cell ERK dynamics. We therefore used CODEX to identify single-cell ERK dynamics patterns under U0126, SL0101 and their combination.

For EGF-stimulated MCF10A WT cells, projection of the CNN features of each ERK trajectory in a tSNE space identified 5 characteristic ERK dynamics, each corresponding to one treatment (Figure 7D-F). Cluster 1 (no EGF stimulation) revealed low frequency ERK activity pulses. Cluster 2 (EGF stimulation) revealed a compact distribution of cells with an initial EGF-triggered ERK peak followed by high frequency ERK pulses. Cluster 3 (EGF, SL0101 treatment) revealed a heterogeneous mix of trajectories, some of which display wider ERK pulses, while others showed lower ERK 
amplitudes. Cluster 4 (EGF, U0126 treatment) revealed a compact distribution of cells with low amplitude ERK pulses. Cluster 5 (EGF, drug combination) revealed a compact cluster distribution of cells with low baseline ERK activity.

For ErbB2 overexpressing MCF10A cells, the t-SNE projection suggested a much higher heterogeneity than for EGF-treated MCF10A WT cells (Figure 7G-I). Cluster 1 (untreated cells) revealed robust ERK pulses. SL0101-mediated RSK inhibition led to a highly heterogeneous landscape of wide, high amplitude ERK pulses (cluster 2), relatively flat ERK trajectories of intermediate amplitude (cluster 3 ) and absence of ERK pulses with low baseline activity (cluster 5). U0126-mediated MEK inhibition led to a compact distribution of cells with low amplitude ERK pulses (cluster 4), as well as some cluster 3 and 5 trajectories. Combination of both drugs shifted most of the population to cluster 5 (absence of ERK pulses and low baseline activity). Thus, targeting MAPK network robustness through RSK inhibition increases ERK heterogeneity, and sensitizes ERK activity to MEK inhibition.

Finally, we evaluated cell cycle dynamics in response to single and combined RSK/MEK inhibition using the G1/S/mitosis geminin-mCherry sensor. MCF10A WT cells treated with $50 \mu \mathrm{M}$ of SL0101, $1 \mu \mathrm{M}$ of U0126 or their combination, were stimulated with $10 \mathrm{ng} / \mathrm{ml}$ EGF and the biosensor activity was followed for 40 hours. Clustering of geminin trajectories from all experiments revealed cell populations with absence of geminin expression (cluster 1), or transient weak geminin expression (cluster 2) assumed not to enter the cell cycle, as well as robust, early (cluster 3), delayed (cluster 4 ) or late (cluster 5) geminin expression, that were assumed to enter the cell cycle (Figure 7J,S7G). We then evaluated the proportion of the geminin profiles for each treatment (Figure 7K). Starved cells mostly showed no geminin expression (cluster 1). EGF stimulation increased cells displaying high geminin expression $(25 \%$ of cells belonging to clusters $3,4,5$ ). RSK inhibition decreased the proportion of cells with high geminin expression and increased the proportion of trajectories in clusters 1 and 2. This most likely occurs because RSK directly regulates several cell-cycle checkpoints (Casalvieri et al., 2017). Weak MEK inhibition using $1 \mu \mathrm{M}$ U0126 led to a mild reduction of cell cycle entry, consistent with moderate effects on ERK dynamics. However, consistently with potent ERK activity inhibition by the combined treatments, cell cycle entry was strongly suppressed. Thus, weakening MAPK robustness through perturbation of the RSK2 negative feedback sensitizes cells to MEK inhibition to potently inhibit ERK activity and shut down cell cycle entry. 

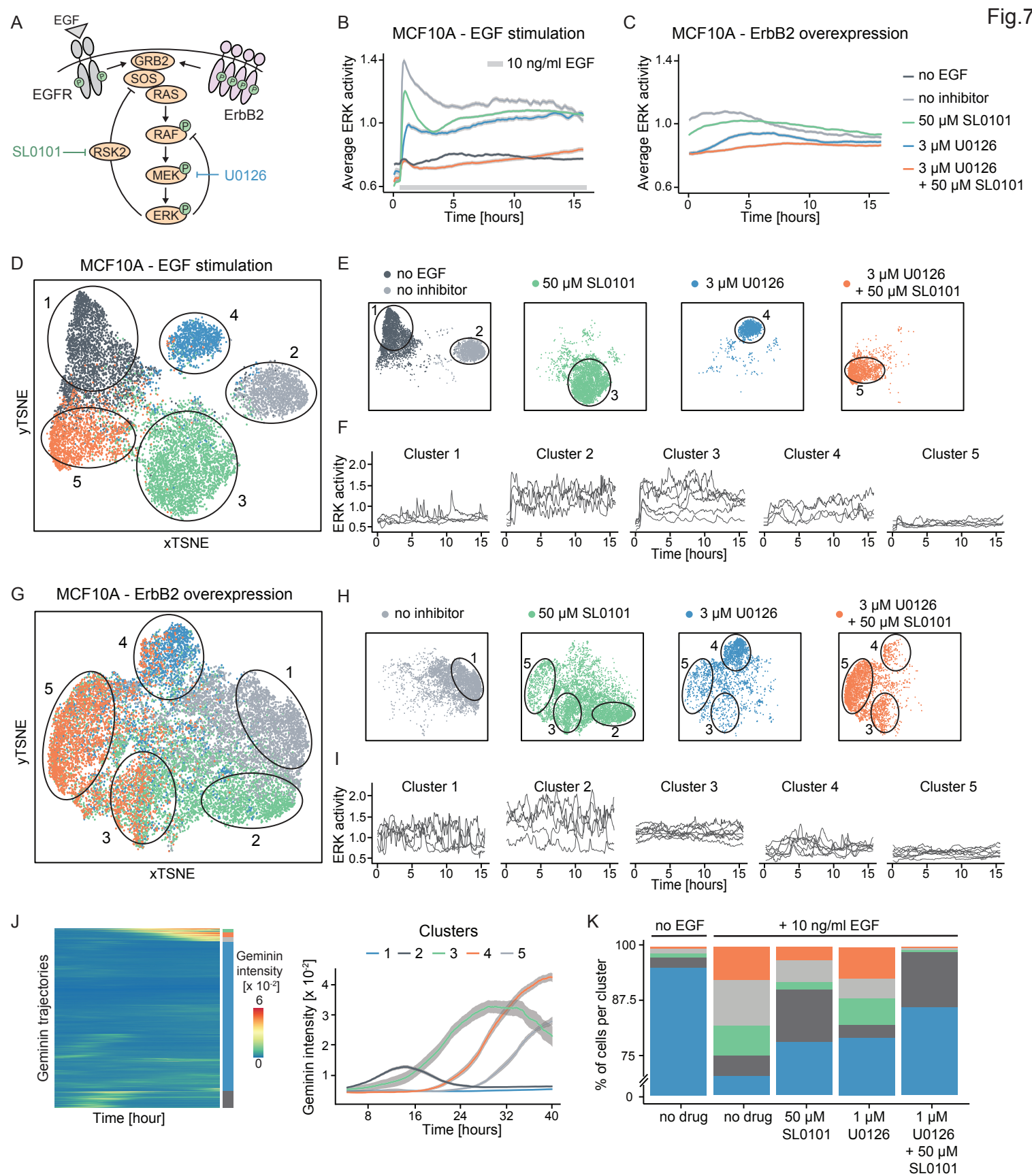

Figure 7: Targeting the RSK2-mediated feedback in combination with MEK inhibition potently inhibits ERK dynamics in response to EGFR and oncogenic ErbB2 inputs. (A) Schematic representation of the MAPK network in response to EGFR inputs in MCF10A WT cells or oncogenic ErbB2 inputs. Cells were treated with single or combined perturbations of RSK (SL0101) and MEK (U0126). (B-C) Average ERK responses evoked in MCF10A WT cells by EGF stimulation $(10 \mathrm{ng} / \mathrm{ml}$ EGF added at $\mathrm{t}=30$ minutes) $(\mathrm{B})$ or ErbB2 overexpressing MCF10A cells $(\mathrm{C})$ under no inhibitor, the RSK (SL0101) and MEK (U0126) inhibitors alone or combined. Shaded areas indicate the 95\% Cl. (DE) tSNE projection of CODEX's CNN features from ERK activity of MCF10A WT cells described in (B). (F) Representative single-cell trajectories for the different clusters displayed in (D-E). (G-H) tSNE projection of CODEX's CNN features from ERK activity of ErbB2 overexpressing cells described in (C). (I) Representative single-cell trajectories for the different clusters displayed in (G-H). (J) Hierarchical clustering (Euclidean distance and Ward D2 linkage) of geminin trajectories in MCF10A WT cells treated with the RSK (SL0101) and MEK (U0126) inhibitors alone or combined ( $N_{\text {noEGF }}=510$ cells, NEGF $=260$, $\left.\mathrm{N}_{\mathrm{sL} 0101}=1190, \mathrm{~N}_{\mathrm{U} 0126}=540, \mathrm{~N}_{\mathrm{sL} 0101+\mathrm{U} 0126}=1740\right)$. The number of clusters was arbitrarily defined to 
bioRxiv preprint doi: https://doi.org/10.1101/2021.07.27.453955; this version posted July 27, 2021. The copyright holder for this preprint (which was not certified by peer review) is the author/funder, who has granted bioRxiv a license to display the preprint in perpetuity. It is made available under aCC-BY-NC-ND 4.0 International license.

separate the different geminin dynamics. Average geminin responses per cluster are displayed on the right. (K) Percentage of geminin trajectories per cluster displayed in $(\mathrm{J})$. The $\mathrm{y}$-axis was truncated for better visualization. 


\section{Discussion}

How the MAPK network is wired to translate different environmental inputs into distinct ERK dynamics, and thus fates, remains poorly understood. This stems from limited experimental throughput to measure single-cell ERK dynamics in response to defined inputs. Our optogenetic circuits overcome this limitation by allowing for automatized dynamic inputs, ERK dynamics measurement in large numbers of cells under multiple perturbations, and for activation of the network at different levels to evaluate the specific properties of subnetworks. Even if optoFGFR does not capture all the properties of endogenous RTKs, our combined modeling and experimental approach showed that it provides valid insights about MAPK network topology downstream of endogenous RTKs (Text S2, Figure S3). Understanding this network topology provides new insight into how to drug network robustness for potent ERK inhibition in an ErbB2-driven oncogenic system.

\section{Negative feedbacks provide robustness to the MAPK network}

What comes strikingly out of our siRNA screens, is that very few perturbations led to penetrant phenotypes (Figure $3 \mathrm{E}$ ). This goes against the intuition that perturbing core nodes of the MAPK network should have a great impact on the downstream ERK activity. This implies that the MAPK network is robust against perturbations of many signaling nodes, warranting high fidelity signal transduction in the presence of heterogeneous node expression levels. This robustness might emerge from functional redundancy of different node isoforms, but also from negative feedbacks, the most notable of which is the classic ERK to RAF negative feedback (Fritsche-Guenther et al., 2011; Sturm et al., 2010).

A striking result of our screen was that the ERK-RSK2-SOS negative feedback operates simultaneously with the aforementioned ERK-RAF feedback in response to the RTK input. The ERK substrate RSK2 has been proposed to mediate negative feedback by disrupting the GRB2-SOS complex (Douville and Downward, 1997; Saha et al., 2012). Our data also suggest that the CRAF and ERK2 isoforms are specifically mediating the ERK-RAF negative feedback. Additional feedbacks have been reported within the MAPK network (Kochańczyk et al., 2017; Lake et al., 2016; Langlois et al., 1995), and even if they have not been highlighted in our screen, they might also shape ERK dynamics. We then hypothesized that these negative feedbacks might warrant robustness against noise in node expression by simultaneously operating at multiple levels in the MAPK network. Bypassing the ERK-RSK2-SOS feedback by optoSOSmediated RAS/ERK subnetwork activation, or by RSK2 perturbation, indicated that the latter is crucial for network robustness and high-fidelity signal transduction. This was apparent from the findings that: 1. ERK dynamics are more sensitive to RNAi perturbations in response to an optoSOS versus an optoFGFR input (Figure 5), and 2. absence of the ERK-RSK2-SOS negative feedback sensitizes the network to additional MEK perturbation (Figure 6). The relative contributions of both feedbacks are difficult to evaluate since in the case of the ERK and RAF, the feedback directly 
involves both nodes. To mechanistically explore the idea that a MAPK network with multiple negative feedbacks provides robustness against noise in the expression level of its components, we tried to model MAPK network topologies with multiple feedbacks. We however were unable to unambiguously identify specific models and parameters while fitting ERK dynamics time-series. The identification of predictive models that take into account the signaling complexity that we have identified will require additional information about the different nodes and their dynamics to further constrain the model topology and parameter space.

\section{Drugging RSK2-mediated network robustness potently shuts down EGFR and oncogenic ErbB2 ERK signaling}

Our genetic circuits, together with system-wide perturbations, indicate that the RTK/MAPK network is robust against node perturbation, which emerges at least in part because of the ERK-RSK2-SOS feedback. We found that this feedback also operates downstream of EGFR and oncogenic ErbB2 signaling in MCF10A cells (Figure 7). In both EGF-treated or ErbB2 overexpressing MCF10A cells, RSK inhibition led to highly heterogeneous ERK dynamics, which resulted in the inability of the MAPK network to cope with network node expression noise. Note that a subset of RSKinhibited cells displayed wider ERK pulses, possibly also indicative of decreased negative feedback (Figure 7F,I). Note that inhibition of RSK2 alone might lead to a highly heterogeneous landscape of ERK dynamics that possibly could promote proliferation/survival in a cell population subset (Gagliardi et al., 2021). A low MEK inhibitor dose mildly affected population-averaged ERK activity. However, combined RSK2/MEK inhibition shifted a large cell population towards robust ERK inhibition in both MCF10A systems. Thus, RSK2 inhibition can be used to drug network robustness, sensitizing the MAPK network to additional perturbations such as MEK inhibition. The ability of this drug combination to efficiently switch off ERK in a large number of cells might mitigate the risk of emergence of drug-resistant cells. We observed that combined RSK2/MEK inhibition also increased cell cycle entry inhibition. Altogether, these results strongly suggest that this does not result from additive effects of both drugs, but through weakening MAPK network robustness by RSK2 inhibition, which then potentiates MEK inhibition. Detailed understanding of MAPK network topology can therefore provide unintuitive insights into efficient pharmacological shut down of ERK signaling.

\section{Additional new insights into regulation of ERK dynamics}

Our screens provided a number of additional insights about MAPK network regulation (Figure 3D,E). PLCG1 KD led to steeper ERK activation and increased oscillatory behavior. Phospholipase $C_{\gamma} 1$ activates calcium signaling, which has itself been shown to regulate RAS/MAPK signaling in a calcium spike frequency-dependent manner (Cullen and Lockyer, 2002; Kupzig et al., 2005). Further investigation will be required to understand the significance of this crosstalk. KD of RAPGEF1, which encodes the Rap1-specific GEF C3G, also led to increased oscillatory ERK behavior. C3G/Rap1 
signaling has previously been shown to collaborate with RAS to regulate NGFdependent sustained ERK dynamics in PC-12 cells (York et al., 1998). Thus, C3G might also fine tune ERK dynamics through a mechanism that remains to be determined. GRB2 KD led to one of the strongest phenotypes with respect to ERK amplitude. GRB2 KD also led to ERK dynamics that fluctuated with distinct kinetics within different cells of the population, and highly heterogeneous ERK amplitudes. GRB2 acts as the RTK-proximal adaptor to activate the RAS-specific SOS (Belov and Mohammadi, 2012; Chardin et al., 1993). As it is operating above SOS, GRB2 lacks a negative feedback that can buffer against heterogeneous expression levels. Low GRB2 levels might therefore be especially prone to inefficient signal transmission from the RTK to the MAPK network. GRB2 might thus be another vulnerable node for drugging network robustness. A similar, although less penetrant phenotype, was observed for KD of FRS2, another adaptor protein directly operating downstream of FGFR (Gotoh, 2008).

With regard to phosphatases, we found that $P P 2 A$ KD led to a robust increased ERK amplitude in response to both optoFGFR and optoSOS inputs (Figure 3D,5A,B). This mostly occurs because protein phosphatase $2 \mathrm{~A}$ is a major phosphatase that can act at multiple levels by dephosphorylating Shc1, MEK1, MEK2, ERK1 and ERK2 (Junttila et al., 2008). In optoFGFR-low PP2A KD cells, oscillatory ERK dynamics displayed increased amplitude (Figure $3 E$ ), suggesting that negative feedback can buffer for loss of phosphatase regulation to some extent. Perturbation of many of the DUSPs known to be expressed in our cell system also led to increased ERK amplitude. These DUSPS consist of classic MAPK phosphatases that localize to the cytosol (DUSP6, DUSP16), the nucleus (DUSP1, DUSP2, DUSP4), or both compartments (DUSP8, DUSP9, DUSP10), as well as of atypical DUSPs (DUSP3, DUSP14, DUSP22, DUSP26) that also can have effects on ERK (Patterson et al., 2009). DUSP6 KD led to increased baseline ERK activity levels (Figure S5C). Consistently, DUSP6 has previously been proposed to pre-emptively dephosphorylate MAPKs to maintain low ERK activity baseline levels at resting state (Huang and Tan, 2012). Our results indicate that perturbation of one DUSP cannot be compensated by the others, suggesting that individual DUSPs regulate specific substrates within the MAPK network. DUSP perturbations affected ERK amplitude directly upon application of the light input. This implies that, at least in our system, DUSP activity does not necessarily require ERKdependent transcription of these phosphatases, as previously observed for DUSP2 that is an ERK-dependent immediate-early gene (Wei et al., 2013). KD of the different DUSPs did not markedly affect oscillatory ERK behavior in optoFGFR-low cells, suggesting that DUSPs are not involved in the MAPK feedback circuitry that operates on timescales of minutes. Finally, DUSP4 KD led to lower ERK amplitude (Figure $3 \mathrm{D}, 5 \mathrm{~A}, \mathrm{~B})$. This might occur because of specificity for other kinases such as $\mathrm{p} 38$ and JNK that might feed back to the MAPK pathway (Patterson et al., 2009).

To sum up, our scalable experimental pipeline for measuring ERK dynamics provides new insights about MAPK network topology, such as novel layers of regulations that will need to be explored in the future, and the characterization of a novel druggable feedback important for MAPK network robustness. This provides new opportunities for 
bioRxiv preprint doi: https://doi.org/10.1101/2021.07.27.453955; this version posted July 27, 2021. The copyright holder for this preprint (which was not certified by peer review) is the author/funder, who has granted bioRxiv a license to display the preprint in perpetuity. It is made available under aCC-BY-NC-ND 4.0 International license.

efficient pharmacological targeting of oncogenic MAPK signaling. With the increasing amount of optogenetic actuators and biosensors available, similar genetic circuits can be designed in the future to study the dynamics of other signaling pathways at scale. 


\section{Material and methods}

\section{Cell culture and reagents}

$\mathrm{NIH} 3 \mathrm{~T} 3$ cells were cultured in DMEM high glucose medium with $5 \%$ fetal bovine serum, $4 \mathrm{mM} \mathrm{L}$-glutamine, $200 \mathrm{U} / \mathrm{ml}$ penicillin and $200 \mu \mathrm{g} / \mathrm{ml}$ streptomycin at $37^{\circ} \mathrm{C}$ with $5 \% \mathrm{CO}_{2}$. All imaging experiments with $\mathrm{NIH} 3 \mathrm{~T} 3$ were done in starving medium consisting of DMEM high glucose supplemented with $0.5 \%$ BSA (Sigma), $200 \mathrm{U} / \mathrm{ml}$ penicillin, $200 \mu \mathrm{g} / \mathrm{ml}$ streptomycin and $4 \mathrm{mM}$ L-Glutamine. MCF10A human mammary cells were cultured in DMEM:F12 supplemented with $5 \%$ horse serum, $20 \mathrm{ng} / \mathrm{ml}$ recombinant human EGF (Peprotech), $10 \mu \mathrm{g} / \mathrm{ml}$ insulin (Sigma), $0.5 \mathrm{mg} / \mathrm{ml}$ hydrocortisone (Sigma), $200 \mathrm{U} / \mathrm{ml}$ penicillin and $200 \mu \mathrm{g} / \mathrm{ml}$ streptomycin. All imaging experiments with MCF10A were done in starving medium consisting in DMEM:F12 supplemented with $0.3 \% \mathrm{BSA}, 0.5 \mathrm{mg} / \mathrm{ml}$ hydrocortisone, $200 \mathrm{U} / \mathrm{ml}$ penicillin and 200 $\mu \mathrm{g} / \mathrm{ml}$ streptomycin. For growth factor stimulations, we used human EGF (AF-100, Peprotech) and human basic FGF (F0291, Sigma). Chemical perturbations were done with SU-5402 (SML0443, Sigma), RAF709 (HY-100510, Lucerna Chem), U0126 (S1102, Selleck chemicals, Lubio), SCH772984 (HY-50846, Lucerna-Chem), SL0101 (559285, Sigma), Cyclosporine A (10-1119, Lucerna-chem) and lonomycin (sc-3592, Santa Cruz). Selection of the cells post transfection was done using Puromycin (P7255, Sigma), Blasticidin S HCl (5502, Tocris) and Hygromycin B (sc-29067, Lab Force).

\section{Plasmids and stable cell line generation}

The optoFGFR construct was a gift from Won Do Heo (Addgene plasmid \# 59776) (Kim et al., 2014). It consists of the myristoylated FGFR1 cytoplasmic region fused with the PHR domain of the cryptochrome2 and tagged with mCitrine. It was cloned in a lentiviral backbone for stable cell line generation. A modified version of the optoFGFR tagged with the red fluorophore mScarlet (Bindels et al., 2017) was cloned in a PiggyBac plasmid pMP-PB (blasticidin resistance). The optoSOS construct is a modified version of the tRFP-SSPB-SOScat-P2A-iLID-CAAX (Addgene plasmid \#86439) (Johnson et al., 2017), in which we replaced the tRFP by mCitrine. The construct was cloned in the pPB3.0.Puro, an improved PiggyBac plasmid generated in our lab with puromycin resistance. The ERK-KTR-mRuby2 and ERK-KTRmTurquoise2 reporters were generated by fusing the ERK Kinase Translocation Reporter (ERK-KTR) (Regot et al., 2014) with mRuby2 (Lam et al., 2012) or mTurquoise2 (Goedhart et al., 2012). The nuclear marker H2B-miRFP703 is a fusion of the human $\mathrm{H} 2 \mathrm{~B}$ clustered histone 11 (H2BC11) with the monomeric near-infrared fluorescent protein miRFP703 (Shcherbakova et al., 2016) (Addgene plasmid \#80001). ERK-KTR-mRuby2, ERK-KTR-mTurquoise2 and H2B-miRFP703 were cloned in the PiggyBac plasmids pPB3.0.Hygro, pSB-HPB (gift of David Hacker, EPFL, (Balasubramanian et al., 2016)) and pPB3.0.Blast, respectively. The S/G2/M biosensor mCherry-hGeminin was generated by fusing mCherry to the ubiquitylation domain of human Geminin (Sakaue-Sawano et al., 2008) and cloned into the 
PiggyBac plasmid pSB-HPB. All constructs in PiggyBac plasmids were co-transfected with the helper plasmid expressing the transposase (Yusa et al., 2011) for stable insertion using the jetPEI (Polyplus) transfection reagent for NIH3T3 cells or FuGene (Promega) transfection reagent for MCF10A cells. After antibiotic selection, NIH3T3 cells were FACS-sorted to generate stable cell lines homogeneously expressing the biosensors. In the case of MCF10A cells, clones with uniform biosensor expression were isolated. To generate ErbB2 overexpressing MCF10A cells, lentiviral transduction using a pHAGE-ERBB2 construct (a gift from Gordon Mills \& Kenneth Scott, Addgene plasmid \#116734) ( $\mathrm{Ng}$ et al., 2018) was performed in the presence of $8 \mathrm{\mu g} / \mathrm{ml}$ polybrene (TR1003, Sigma) in cells already expressing H2B-miRFP703 and ERK-KTR-mTurquoise2. Cells were further selected with $5 \mu \mathrm{g} / \mathrm{ml}$ puromycin.

\section{Live imaging of ERK dynamics}

NIH3T3 cells were seeded in 96 well 1.5 glass bottom plates (Cellvis) coated with 10 $\mu \mathrm{g} / \mathrm{ml}$ Fibronectin (Huber lab) using $1.5 \times 10^{3}$ cells/well and incubated for 24 hours. MCF10A cells were seeded in 24-well 1.5 glass bottom plates (Cellvis) coated with 5 $\mu \mathrm{g} / \mathrm{ml}$ Fibronectin (Huber lab) at $1 \times 10^{5}$ cells/well and incubated for 48 hours. NIH3T3 cells were washed with PBS and incubated in starving medium for 4 hours in the dark before starting the experiment. MCF10A cells were starved for 7 hours (ERK dynamics measurements) or at least $24 \mathrm{~h}$ hours (geminin dynamics measurements) before starting the experiments. In experiments involving drug perturbations, cells were incubated for 2 hours (or 1 hour in MCF10A experiments) with the inhibitor(s). Imaging was performed with an epifluorescence Eclipse Ti inverted fluorescence microscope (Nikon) using a Plan Apo air 20x (NA 0.8) objective. Nikon Perfect Focus System (PFS) was used to keep cells in focus throughout the experiment. Illumination was done with a SPECTRA $X$ light engine (Lumencor) with the following filters (Chroma): mTurquoise2: 440 nm LED, 470lp, 69308 CFP/YFP/mCherry-ET, CFP 458-482; mCitrine: 508 nm LED, ET500/20x, 69308bs, ET535/30m; mRuby2 and mCherry: 555 nm LED, ET575/25x, 69008bs, 59022m, miRFP703: 640 nm LED, ET640/30x, 89100 bs Sedat Quad, 84101m Quad. Images were acquired with an Andor Zyla 4.2 plus camera at a 16-bit depth. Image acquisition and optogenetic stimulation were controlled with the NIS-Element JOBS module. For NIH3T3 experiments, ERK-KTRmRuby2 and H2B-miRFP703 were acquired at 1-minute interval and $470 \mathrm{~nm}$ light inputs were delivered at specific frequencies and intensities (see below). MCF10A image acquisition was performed at 5-minute time resolution. Growth factor stimulations were done by manually pipetting EGF and bFGF during the experiment. We used mCitrine intensity to quantify the expression level of the optogenetic constructs. However, as mCitrine excitation leads to optoFGFR or optoSOS activation, we acquired one frame with the ERK-KTR-mRuby2, the H2B-miRFP703 and the mCitrine-tagged optoFGFR or optoSOS only at the end of each NIH3T3 experiments. All experiments were carried on at $37^{\circ} \mathrm{C}$ with $5 \% \mathrm{CO}_{2}$. 


\section{Optogenetic stimulation}

Light stimulations were delivered with a $470 \mathrm{~nm}$ LED light source that was hardwaretriggered by the camera to generate light pulses of reproducible duration. Light stimulations of defined intensity and duration were programmed to be automatically delivered at specific timepoints. To define the dose of light received by the cells, we measured the $470 \mathrm{~nm}$ light intensity at the focal plane using an optical power meter (X-Cite Power Meter, Lumen Dynamics Group) and converted this value to a power density as

$$
\text { Light power density }=\text { Light intensity } \times \frac{1}{\pi \times\left(\frac{F N}{2 \times \text { Magnification }}\right)^{2}}\left[\frac{\mathrm{mW}}{\mathrm{cm}^{2}}\right]
$$

with $\mathrm{FN}=18 \mathrm{~mm}$. The obtained value was then multiplied by the duration of the pulse to obtain the dose of light received by the cells for each light pulse.

$$
\text { Light dose }=\text { Light power density } \times \text { Pulse duration }=\left[\frac{\mathrm{mW} \times s}{\mathrm{~cm}^{2}}\right]=\left[\frac{\mathrm{mJ}}{\mathrm{cm}^{2}}\right]
$$

For stimulation of the optoFGFR cells, the $470 \mathrm{~nm}$ LED intensity was limited to a low dose by combining a ZET470/10x filter and a ND filter 5\% (Chroma). Transient stimulations were done with a single pulse, while sustained stimulations were done with single pulses delivered every 2 minutes. For stimulation of the optoSOS cells, we used the $470 \mathrm{~nm}$ LED with a ET470/24x filter (no ND filter). Transient stimulations were done with 5 pulses repeated at 20-second intervals, while sustained stimulations were done using 5 pulses of light repeated at 20 -second intervals, delivered every 2 minutes.

\begin{tabular}{|l|l|l|l|l|l|}
\hline Figures & System & $\begin{array}{l}\text { Power } \\
\text { density }\end{array}$ & Pulse duration & Dose & $\begin{array}{l}\text { Stimulation } \\
\text { pattern }\end{array}$ \\
\hline $\begin{array}{l}\text { 1B-C, } \\
\text { S1A,S1D }\end{array}$ & optoFGFR & $180 \mathrm{~mW} / \mathrm{cm}^{2}$ & $100 \mathrm{~ms}$ & $18 \mathrm{~mJ} / \mathrm{cm}^{2}$ & transient \\
\hline S1B-C & $\begin{array}{l}\text { optoFGFR } \\
\text { (mScarlet) }\end{array}$ & $>180 \mathrm{~mW} / \mathrm{cm}^{2}$ & $100 \mathrm{~ms}$ & $>18 \mathrm{~mJ} / \mathrm{cm}^{2}$ & transient \\
\hline 2A-B & optoFGFR & variable & variable & variable & transient \\
\hline 2C & optoFGFR & $180 \mathrm{~mW} / \mathrm{cm}^{2}$ & $100 \mathrm{~ms}$ & $18 \mathrm{~mJ} / \mathrm{cm}^{2}$ & variable \\
\hline $\begin{array}{l}\text { 2D- } \\
\text { E,S2E,S3 } \\
\text { B,S3K,3B, } \\
\text { 3D-E,S4C- } \\
\begin{array}{l}\text { D,S5A- } \\
\text { D,4E,5B,6 }\end{array}\end{array}$ & optoFGFR & $180 \mathrm{~mW} / \mathrm{cm}^{2}$ & $100 \mathrm{~ms}$ & $18 \mathrm{~mJ} / \mathrm{cm}^{2}$ & sustained \\
\hline
\end{tabular}




\begin{tabular}{|l|l|l|l|l|l|}
\hline $\begin{array}{l}\text { A-H,S7A- } \\
\text { D }\end{array}$ & & & & & \\
\hline $2 \mathrm{~F}$ & optoFGFR & variable & $100 \mathrm{~ms}$ & variable & sustained \\
\hline $4 \mathrm{~B}$ & optoSOS & $6 \mathrm{~W} / \mathrm{cm}^{2}$ & $\begin{array}{l}\text { variable } \times 100 \\
\mathrm{~ms}(20 \mathrm{sec} \\
\text { interval })\end{array}$ & $0.6 \mathrm{~J} / \mathrm{cm}^{2}$ & transient \\
\hline S6A & optoSOS & variable & $\begin{array}{l}\text { variable } \mathrm{x} \\
100 \mathrm{~ms}(20-\mathrm{sec} \\
\text { interval })\end{array}$ & variable & transient \\
\hline 4C & optoSOS & $6 \mathrm{~W} / \mathrm{cm}^{2}$ & $\begin{array}{l}5 \times 100 \mathrm{~ms}(20- \\
\text { sec interval })\end{array}$ & $0.6 \mathrm{~J} / \mathrm{cm}^{2}$ & variable \\
\hline 4D & optoSOS & variable & $\begin{array}{l}5 \times 100 \mathrm{~ms}(20- \\
\text { sec interval })\end{array}$ & variable & sustained \\
\hline $\begin{array}{l}\text { 4E,S6B,S6 } \\
\text { C-D,5A- } \\
\text { C,6A-D }\end{array}$ & optoSOS & $6 \mathrm{~W} / \mathrm{cm}^{2}$ & $\begin{array}{l}5 \times 100 \mathrm{~ms}(20- \\
\text { sec interval })\end{array}$ & $0.6 \mathrm{~J} / \mathrm{cm}^{2}$ & sustained \\
\hline
\end{tabular}

\section{TIRF imaging of optoFGFR dynamics}

Cells were seeded at a density of $1 \times 10^{3}$ per well in 96 well 1.5 glass bottom plates (Cellvis) coated with $10 \mu \mathrm{g} / \mathrm{ml}$ Fibronectin (Huber lab) and incubated for 24 hours at $37^{\circ} \mathrm{C}$ with $5 \% \mathrm{CO}_{2}$. Before imaging, cells were washed with PBS and incubated in starving medium for 4 hours in the dark. Imaging was performed with an epifluorescence Eclipse $\mathrm{Ti}$ inverted fluorescence microscope (Nikon) using a CFI Apochromat TIRF 100x oil (NA 1.49). Images were acquired with an Andor Zyla 4.2 plus camera at a 16-bit depth. TIRF images were acquired with a $561 \mathrm{~nm}$ laser using a ET575/25 filter in front of the ZT488/561rpc (Chroma) to prevent nonspecific activation of the CRY2. MetaMorph software (Universal Imaging) was used for acquisition. TIRF images of the optoFGFR-mScarlet were acquired at a 20-second interval. Optogenetic stimulation was done using a $470 \mathrm{~nm}$ LED (SPECTRA X, Lumencor) (Figure S1B-C). All experiments were carried on at $37^{\circ} \mathrm{C}$ with $5 \% \mathrm{CO}_{2}$.

\section{Image processing pipeline}

Nuclear segmentation was done in CellProfiler 3.0 (McQuin et al., 2018) using a threshold-based approach of the H2B channel. In the case of MCF10A cells, nuclear segmentation was preceded by prediction of nuclear probability using a random forest classifier based on different pixel features available in llastik software (Berg et al., 2019). To measure the ERK-KTR fluorescence in the cytosol, the nuclear mask was first expanded by 2 pixels to exclude the blurred edges of the nucleus. The new mask was then further expanded by 4 pixels in a threshold-based manner in order to obtain a "ring" area corresponding to the cytoplasmic ERK-KTR. ERK activity was obtained by calculating the ratio between the average cytosolic pixel intensity and the average 
nuclear pixel intensity. Single-cell tracking was done on nuclear centroids with MATLAB using $\mu$-track 2.2.1 (Jaqaman et al., 2008). The final images containing the ERK-KTR-mRuby2, H2B-miRFP703 and the optoFGFR-mCitrine (or optoSOS$\mathrm{mCitrine)}$ channels were processed using the same CellProfiler settings as the time lapse images. Intensity of the mCitrine was extracted using the ERK-KTR cytoplasmic mask and used to classify cells into low or high expressors in a threshold-based manner. For optoFGFR-evoked ERK responses, the threshold was defined empirically to separate oscillatory and non-oscillatory ERK responses (low $<-1.75$ (log10 mCitrine intensity) < high). For optoSOS-evoked ERK responses, the threshold was defined empirically to separate cells with low or high ERK response amplitudes (low $<-1.25$ (log10 mCitrine intensity) < high). The same thresholds were kept across experiments to compare low and high expressors.

The optoFGFR-mScarlet dimers/oligomers were segmented using the pixel classification module from llastik (Berg et al., 2019). OptoFGFR dimers, cell background and trafficking vesicles were manually annotated on images before and after the light stimulation. A probability map of the optoFGFR dimers classification was exported as TIFF for each frame. We then computed the mean of pixel intensities from the binarized mask obtained with Ilastik using Fiji (Figure S1B,C).

\section{Quantification of ERK activity and cell-cycle}

We wrote a set of custom $R$ scripts to automatically calculate the ERK-KTR cytoplasmic/nuclear ratio as a proxy for ERK activity for each single-cell, link singlecell ERK responses with the corresponding optoFGFR/optoSOS intensity value and export the corresponding ERK single-cell trajectories. For NIH3T3 data, outliers in ERK single-cell trajectories were removed using a clustering-based approach (https://github.com/pertzlab/Outlier app). Trajectories with an ERK-KTR cytoplasmic/nuclear ratio higher than 0.8 or lower than 0.2 before stimulation, above 1.6 during the whole experiment or displaying single time point spiking values were removed. For MCF10A data, trajectories with an ERK-KTR cytoplasmic/nuclear ratio above 2 or shorter than $90 \%$ of the total experiment duration were removed. All the $R$ codes used for further analysis are available as supplementary information. Hierarchical clustering analysis of single-cell trajectories (Figure 2D,S5F,G,S6B,7J,K) was done using Time Course Inspector (Dobrzyński et al., 2019).

\section{Modeling}

The model for the EGF and light stimulated ERK cascade is a kinetic model, representing the EGF receptor, the inter-cellular proteins (RAS, RAF, MEK, ERK) as well as a negative feedback from ERK to RAF and the inactivation of the EGF receptor in the form of endocytosis (Figure S3A). We explicitly modelled the ERK-KTR readout through nuclear and cytosolic KTR. The initial fraction of cytosolic KTR is estimated from the data through the parameter $k t r_{\text {init }}$. The KTR readout $Y(t)$ was taken to be the ratio of cytosolic KTR over nuclear KTR with additive Gaussian noise 


$$
\begin{gathered}
Y(t)=\frac{K T R}{K T R^{*}}+\epsilon \\
\epsilon \sim \operatorname{Normal}\left(0, \sigma^{2}\right)
\end{gathered}
$$

where the variance of the measurement noise $\sigma^{2}$ was estimated from the data.

Table S1 shows all modelled species, their notation used for the equation, as well as the initial values. We assume that in the beginning of the experiment, all species are in the inactive form, reflecting the fact that the cells have been starved. The total concentrations of all species have been normalized to 1 . The model equations are shown in Table S2. The phosphorylation events are modelled with Michaelis-Menten kinetics. The negative feedback is modelled through the modeling species $N F B$ and its "active" version $N F B^{*}$ which affects the dephosphorylation rate of $R A F$ linearly. The activation, endocytosis and recycling of the EGF receptor is modelled linearly. The model parameters are described in Table S3. For the modeling of the two smaller models (without feedback (Figure S3E) or without endocytosis (Figure S3H)), we set the corresponding parameters $\left(k_{n f b}\right.$ and $\left.r_{2,3}\right)$ to zero.

For the parameter inference we used a Nested Sampling algorithm as described in (Mikelson and Khammash, 2020). The inference was performed on the ETH Highperformance Cluster Euler and was done using the parallel implementation on 48 cores. The algorithm was run for 24 hours or until the algorithm stopped because the termination criterion $\Delta_{L F N S}$ (see (Mikelson and Khammash, 2020) for details) was $-\infty$. As prior distributions we chose for all parameters non-informative log-uniform priors between $10^{-5}$ and $10^{5}$, except for $k t r_{\text {init }}$ for which we chose a uniform prior on the interval $[0,1]$ and for $\sigma$ for which we chose a log-uniform prior between $10^{-5}$ and 1 . Predictive distributions can be found on Figure S3C,D,F,G,I,J.

\section{RNAi perturbation screen}

We used Ingenuity Pathway Analysis (IPA, Qiagen) to select proteins directly interacting with ERK, MEK, RAF, RAS and FGFR, that are known to be expressed in NIH3T3 cells using a proteomics approach (Jensen et al., 2009; Schwanhäusser et al., 2011) (Table S4). We then imported this protein list in STRING (Jensen et al., 2009) to generate an interaction network with a minimum interaction score of 0.4 (Figure 3A). We targeted these selected proteins with RNA interference, using the siPOOL technology (one siPOOL containing a mix of 30 siRNAs targeting the same gene (Hannus et al., 2014), sequences available in supplementary). We arranged the siPOOLs in a 96 well plate format (in columns 2-5 and 8-11, one well per siPOOL) with the non-targeting siRNA (CTRL) and the positive control (mix of $5 \mathrm{nM}$ siPOOL against $E R K 1$ and $5 \mathrm{nM}$ siPOOL against ERK2) placed alternately in columns 1, 6, 7 and 12. Cells were reverse transfected using RNAiMAX (Thermofischer, 13778150) following the recommended siPOOL transfection protocol. OptoFGFR-expressing cells were transfected with $10 \mathrm{nM}$ of siPOOL in a 96 well 1.5 glass bottom plate (Cellvis) coated with $10 \mu \mathrm{g} / \mathrm{ml}$ Fibronectin (Huber Lab) at $0.3 \times 10^{3}$ cells/well density and incubated for 72 hours at $37^{\circ} \mathrm{C}$ and $5 \% \mathrm{CO}_{2}$. For the imaging, the 96 well plate was divided into 15 
sub-experiments, each sub-experiment consisting of a negative control well, a positive control well and 4 wells with different siPOOLs. We selected 2 FOVs per well and programmed the microscope to run the 15 experiments sequentially, acquiring the ERK-KTR-mRuby2 and the H2B-miRFP703 channels with a 1-minute interval, stimulating the cells with sustained optoFGFR inputs (2-minute intervals, $18 \mathrm{~mJ} / \mathrm{cm}^{2}$ ), and acquiring a final frame with ERK-KTR-mRuby2, H2B-miRFP703 and optoFGFR$m$ Citrine (Figure 3B,D,E,S4C,D,S5A-D,5B,6E-H). For the optoSOS system, we limited the perturbation screen to targets acting below RAS (Figure 5A-C,S6C,D). Stimulations were done with sustained optoSOS inputs (2-minute intervals, $5 \times 0.6$ $\mathrm{J} / \mathrm{cm}^{2}$ ). For EGF stimulation, cells were stimulated with $1 \mathrm{ng} / \mathrm{ml}$ EGF at $\mathrm{t}=5$ minutes (Figure S5E-G).

\section{Real-time $q P C R$}

Cells were transfected with different concentrations of siPOOL in a 24 well plate at 5 $\times 10^{3}$ cells/well density and incubated at $37^{\circ} \mathrm{C}$ with $5 \% \mathrm{CO}_{2}$ for 72 hours before RNA isolation. Reverse transcription was done with the ProtoScript II reverse transcriptase kit (Bioconcept, M0368L). Real-time qPCR reactions were run using the MESA Green pPCR MasterMix Plus for SYBR Green assay (Eurogenetec, RT-SY2X-03+WOU) on the Rotor-Gen $Q$ device (Qiagen). Each sample was tested in triplicate. Expression level of the gene of interest was calculated using the $2^{-\Delta \triangle C t}$ method with $G A P D H$ expression level as internal control (Figure S4A). The following primers were used for the RT-qPCR reaction (designed with the Real-time PCR (TaqMan) Primer and Probes Design Tool from GenScript).

\begin{tabular}{|l|l|l|}
\hline Target & Forward sequences & Reverse sequences \\
\hline ERK1 & 5'-GGTTGTTCCCAAATGCTGACT-3' & 5'-CAACTTCAATCCTCTTGTGAGGG-3' \\
\hline ERK2 & 5'-TCCGCCATGAGAATGTTATAGGC-3' & 5'-GGTGGTGTTGATAAGCAGATTGG-3' \\
\hline MEK1 & 5'-AAGGTGGGGGAACTGAAGGAT-3' & 5'-CGGATTGCGGGTTTGATCTC-3' \\
\hline MEK2 & 5'-GTTACCGGCACTCACTATCAA C-3' & 5'-CCTCCAGCCGCTTCCTTTG-3' \\
\hline GAPDH & 5'-ACCCAGAAGACTGTGGATGG-3' & 5'-TCAGCTCAGGGATGACCTTG-3' \\
\hline
\end{tabular}

\section{Immunoblotting}

Cells were transfected with $10 \mathrm{nM}$ siPOOL in 6 well plates at $6 \times 10^{4}$ cells/well density and incubated at $37^{\circ} \mathrm{C}$ with $5 \% \mathrm{CO}_{2}$ for 72 hours. Cells were lysed in a buffer containing $10 \mathrm{mM}$ Tris $\mathrm{HCl}, 1 \mathrm{mM}$ EDTA and 1\% SDS. Protein concentration was determined with the $\mathrm{BCA}^{\mathrm{TM}}$ protein assay kit (ThermoFisher, 23227). Home cast $10 \%$ SDS gels or Novex 4\%-20\% 10 well Mini Gels (Thermofisher, XP04200) were used for SDS page. Transfer was done using PVDF membranes and a Trans-Blot SD SemiDry Electrophoretic Transfer Cell (Bio-Rad). Imaging was done with an Odyssey Fluorescence scanner (Li-COR) (Figure 3C, S4B). The following primary antibodies 
were used: anti-total ERK (M7927, Sigma), anti-MEK1 (ab32091, Abcam), anti-MEK2 (ab32517, Abcam), anti-BRAF (sc-5284, Santa Cruz), anti-CRAF (9422S, Cell Signaling Technology), anti-SOS1 (610096, Biosciences), anti-GRB2 (PA5-17692, Invitrogen). Anti-GAPDH (sc-32233, Santa Cruz) was used as protein of reference. For the secondary antibodies, we used the IRDye 680LT donkey anti-mouse IgG (92668022, Li-COR), IRDye $800 \mathrm{CW}$ goat anti-mouse (926-32210, Li-COR) and IRDye $800 \mathrm{CW}$ donkey anti-rabbit (926-32213, Li-COR). Protein detection was done with the Image Studio ${ }^{\text {TM }}$ Lite software.

\section{Time-series feature extraction}

We used custom scripts to extract features of ERK responses to transient optogenetic inputs (Figure 2B,S6A) and to sustained GF inputs (Figure S2B,D). The maximum peak (maxPeak) is the absolute value of the highest ERK activity in the trajectory. To estimate the full width at half maximum (FWHM), we first removed the baseline of the trajectories and increased their sampling frequency by a factor 30 with spline interpolation. On the resulting trajectory, we applied a "walk" procedure to quantify the FWHM. In this method, a pointer walks left and right (i.e. opposite and along the direction of time respectively) from the maximum point of the trajectory. The pointer stops whenever the half maximum value is crossed. Both stops define a left and a right border, the time difference between these 2-border time-points gives the FWHM. To avoid reporting aberrant FWHM values in cases where a peak cannot be clearly defined, we excluded FWHM calculation for trajectories where the fold change between the baseline (mean activity before stimulation) and the maximum value of the trajectory was below a threshold manually defined.

To evaluate ERK amplitude in response to sustained high optoFGFR or optoSOS inputs (Figure 3D,4E,5A,B), we extracted the maxPeak from a fixed number of singlecell ERK trajectories within a 10 minutes time window following the start of the stimulation. The maxPeak value was plotted as violin plots for each condition. For the comparison of both optogenetic systems (Figure 4E), statistical analysis was done by comparing low and high expressing cells within and across optogenetic systems with a Wilcoxon test using the FDR p-value correction $\left({ }^{*}<0.05,{ }^{* *}<0.005,{ }^{* * *}<0.0005\right.$, $* * * *<0.00005$ ). For the RNAi perturbations (Figure $3 \mathrm{D}, 5 \mathrm{~A}$ ), statistical analysis was done for each optogenetic system by comparing each perturbation to the negative control (CTRL) with a Wilcoxon test using the FDR p-value correction $\left({ }^{*}<0.05\right.$, $\left.{ }^{* *}<0.005,{ }^{* * *}<0.0005,{ }^{* * * *}<0.00005\right)$. The obtained maxPeak for each siRNA perturbation was then z-scored to the non-targeting siRNA (CTRL) (Figure 5B).

To quantify the efficiency of the MEK inhibitor (U0126) on the reduction of ERK activity under sustained high optoFGFR or optoSOS inputs, extraction of the maxPeak was limited by the fact that several U0126 concentrations led to a full suppression of ERK amplitudes. Therefore, we extracted ERK amplitudes at a fixed time point ( $\mathrm{t}_{\text {fixed optoFGFR }}$ $=10$ minutes, $t_{\text {fixed }}$ optosos $=7$ minutes), which correspond to the maximal ERK amplitudes in the control condition (no U0126). The obtained ERK amplitudes were then plotted per U0126 concentration (Figure 6B,F,S7B). The spreading of ERK amplitude at the fixed time point was evaluated by computing the standard deviation 
of the extracted ERK activity per condition (Figure 6C,G,S7C). The IC50 was calculated by fitting a Hill function to the mean ERK activity of each concentration (Figure 6D,H,S7D).

\section{Identification of ERK dynamics phenotypes using CODEX}

We first trained a convolutional neural network (CNN) to classify input ERK trajectories into any of the siRNA-perturbed conditions (Figure S5A). For this purpose, we used a CNN architecture composed of 4 1D-convolution layers with 20 kernels of size 5, followed by a convolution layer with 20 kernels of size 3 and one layer of 10 kernels of size 3 . The responses are then pooled with global average pooling to generate a vector of 10 features that is passed to a $(10,63)$ fully connected layer for classification. Each convolutional layer is followed by ReLU and batch normalization. The CNN was trained to minimize the cross-entropy loss, with $L 2$ weight penalty of $1 e^{-3}$.

In order to identify siRNA treatments that induced a distinctive phenotype, we selected the 10 conditions for which the CNN classification precision was the highest on the validation set (Table S4, "CODEX accuracy"). To these 10 conditions, we also added the negative control (non-targeting siRNA (CTRL)). We trained a second CNN, with the same architecture and training parameters, but limited to recognizing the 11 selected treatments in order to obtain a clear embedding of these hits. With this new model, we extracted the features used for the classification of the trajectories (i.e. the input representation after the last convolution layer) and projected them with tSNE (Python's sklearn implementation, perplexity of 100, learning rate of 600 and 2500 iterations) (Figure S5B). We selected 10 prototype curves for each treatment by taking the trajectories for which the second CNN's classification confidence (i.e. the probability for the actual class of the inputs) were the highest in the validation set (Figure 3E).

To visualize the ERK dynamics landscape in MCF10A WT cells and in MCF10A cells with ErbB2 overexpression, we trained one CNN for each cell line. These CNNs were trained to recognize the drug treatment applied on cells, using single-cell ERK traces as input. The architecture of the CNNs is the same as described previously. The only difference lies in the number of outputs in the final fully connected layer, which were set to the number of drug treatments. Features used for the classification of the trajectories were then projected with tSNE (Figure 7D,E,G,H). Clusters were manually defined in regions of the tSNE with a high density of points. We used the interactive CODEX application to visualize the trajectories associated with each cluster and manually selected representative trajectories (Figure 7F,I).

\section{Peak detection and classification of oscillatory trajectories}

The number of ERK activity peaks was calculated with a custom algorithm that detects local maxima in time series. First, we applied a short median filter to smoothen the data with a window width of 3 time points. Then, we ran a long median filter to estimate the long-term bias with a window width of 15 time points. This bias was then subtracted from the smoothed time series and we only kept the positive values. If no point in this processed trajectory was exceeding a manual threshold of 0.075 , all variations were 
considered as noise and no peak was extracted from the trajectory. The remaining trajectories were then rescaled to $[0,1]$. Finally, peaks were detected as points that exceeded a threshold which was manually set to 0.1 . Peaks that were found before the first stimulation or after the last stimulation were filtered out.

The classification of trajectories into oscillatory and non-oscillatory behaviors was performed after the peak detection step. Cells were called oscillatory if at least 3 peaks were detected with the peak detection procedure (Figure S5D). Statistical analysis was done using a pairwise t-test comparing each perturbation to the control for high and low levels of optoFGFR independently, with FDR p-value correction $\left({ }^{*}<0.05\right.$, $\left.{ }^{* *}<0.005,{ }^{* * *}<0.0005,{ }^{* * * *}<0.00005\right)$.

\section{Acknowledgement}

This work was supported by SystemsX.ch, Swiss Cancer League and Swiss National Science Foundation grants to Olivier Pertz and by the European Union's Horizon 2020 and innovation program under grant agreement No. 730964 (TRANSVAC project) to Mustafa Khammash. We thank Won Do Heo for sharing the optoFGFR plasmid and David Hacker for sharing the pSB-HPB plasmid. We thank the Microscopy Imaging Center of the University of Bern for its support.

\section{Authors contribution}

O.P. and C.D. designed the study. C.D. developed the optogenetic systems and imaging pipelines. CD performed the experiment and image analysis on NIH3T3. A.F and P.A.G. performed the experiments and image analysis on MCF10A. M.D. developed the processing pipelines. C.D processed the data. C.D., M.-A.J., A.F and P.A.G. analyzed the data. M.-A.J. conducted the CNN analysis. J.M. performed mathematical modeling. O.P and M.K. supervised the work. O.P., C.D. and J.M. wrote the paper.

\section{Conflict of interest}

The authors declare having no conflict of interest. 


\section{Bibliography}

Albeck, J.G., Mills, G.B., and Brugge, J.S. (2013). Frequency-modulated pulses of ERK activity transmit quantitative proliferation signals. Mol. Cell 49, 249-261.

Arkun, Y., and Yasemi, M. (2018). Dynamics and control of the ERK signaling pathway: Sensitivity, bistability, and oscillations. PLoS ONE 13, e0195513.

Arteaga, C.L., and Engelman, J.A. (2014). ERBB receptors: from oncogene discovery to basic science to mechanism-based cancer therapeutics. Cancer Cell 25, 282-303.

Avraham, R., and Yarden, Y. (2011). Feedback regulation of EGFR signalling: decision making by early and delayed loops. Nat. Rev. Mol. Cell Biol. 12, 104-117.

Balasubramanian, S., Wurm, F.M., and Hacker, D.L. (2016). Multigene expression in stable $\mathrm{CHO}$ cell pools generated with the piggyBac transposon system. Biotechnol. Prog. 32, 1308-1317.

Belov, A.A., and Mohammadi, M. (2012). Grb2, a double-edged sword of receptor tyrosine kinase signaling. Sci. Signal. 5, pe49.

Benedetti, L., Barentine, A.E.S., Messa, M., Wheeler, H., Bewersdorf, J., and De Camilli, P. (2018). Light-activated protein interaction with high spatial subcellular confinement. Proc Natl Acad Sci USA 115, E2238-E2245.

Berg, S., Kutra, D., Kroeger, T., Straehle, C.N., Kausler, B.X., Haubold, C., Schiegg, M., Ales, J., Beier, T., Rudy, M., et al. (2019). ilastik: interactive machine learning for (bio)image analysis. Nat. Methods 16, 1226-1232.

Bindels, D.S., Haarbosch, L., van Weeren, L., Postma, M., Wiese, K.E., Mastop, M., Aumonier, S., Gotthard, G., Royant, A., Hink, M.A., et al. (2017). mScarlet: a bright monomeric red fluorescent protein for cellular imaging. Nat. Methods 14, 53-56.

Blum, Y., Mikelson, J., Dobrzyński, M., Ryu, H., Jacques, M.-A., Jeon, N.L., Khammash, M., and Pertz, O. (2019). Temporal perturbation of ERK dynamics reveals network architecture of FGF2/MAPK signaling. Mol. Syst. Biol. 15, e8947.

Blüthgen, N., and Legewie, S. (2013). Robustness of signal transduction pathways. Cell. Mol. Life Sci. 70, 2259-2269.

Casalvieri, K.A., Matheson, C.J., Backos, D.S., and Reigan, P. (2017). Selective targeting of RSK isoforms in cancer. Trends Cancer 3, 302-312.

Chardin, P., Camonis, J.H., Gale, N.W., van Aelst, L., Schlessinger, J., Wigler, M.H., and Bar-Sagi, D. (1993). Human Sos1: a guanine nucleotide exchange factor for Ras that binds to GRB2. Science 260, 1338-1343.

Cherfils, J., and Zeghouf, M. (2013). Regulation of small GTPases by GEFs, GAPs, and GDls. Physiol. Rev. 93, 269-309.

Cullen, P.J., and Lockyer, P.J. (2002). Integration of calcium and Ras signalling. Nat. Rev. Mol. Cell Biol. 3, 339-348.

Dobrzyński, M., Jacques, M.-A., and Pertz, O. (2019). Mining single-cell time-series datasets with Time Course Inspector. Bioinformatics.

Douville, E., and Downward, J. (1997). EGF induced SOS phosphorylation in PC12 cells 
involves P90 RSK-2. Oncogene 15, 373-383.

Duan, L., Hope, J., Ong, Q., Lou, H.-Y., Kim, N., McCarthy, C., Acero, V., Lin, M.Z., and Cui, B. (2017). Understanding CRY2 interactions for optical control of intracellular signaling. Nat. Commun. 8, 547.

Erickson, K.E., Rukhlenko, O.S., Posner, R.G., Hlavacek, W.S., and Kholodenko, B.N. (2019). New insights into RAS biology reinvigorate interest in mathematical modeling of RAS signaling. Semin. Cancer Biol. 54, 162-173.

Ferrell, J.E., and Bhatt, R.R. (1997). Mechanistic studies of the dual phosphorylation of mitogen-activated protein kinase. J. Biol. Chem. 272, 19008-19016.

Fritsche-Guenther, R., Witzel, F., Sieber, A., Herr, R., Schmidt, N., Braun, S., Brummer, T., Sers, C., and Blüthgen, N. (2011). Strong negative feedback from Erk to Raf confers robustness to MAPK signalling. Mol. Syst. Biol. 7, 489.

Gagliardi, P.A., Dobrzyński, M., Jacques, M.A., Dessauges, C., Ender, P., Blum, Y., Hughes, R.M., Cohen, A.R., and Pertz, O. (2021). Collective ERK/Akt activity waves orchestrate epithelial homeostasis by driving apoptosis-induced survival. Dev. Cell 56, 1712-1726.e6.

Goedhart, J., von Stetten, D., Noirclerc-Savoye, M., Lelimousin, M., Joosen, L., Hink, M.A., van Weeren, L., Gadella, T.W.J., and Royant, A. (2012). Structure-guided evolution of cyan fluorescent proteins towards a quantum yield of $93 \%$. Nat. Commun. 3, 751.

Gotoh, N. (2008). Regulation of growth factor signaling by FRS2 family docking/scaffold adaptor proteins. Cancer Sci. 99, 1319-1325.

Hannus, M., Beitzinger, M., Engelmann, J.C., Weickert, M.-T., Spang, R., Hannus, S., and Meister, G. (2014). siPools: highly complex but accurately defined siRNA pools eliminate offtarget effects. Nucleic Acids Res. 42, 8049-8061.

Huang, C.-Y., and Tan, T.-H. (2012). DUSPs, to MAP kinases and beyond. Cell Biosci. 2, 24.

Jacques, M.A., Dobrzyński, M., Gagliardi, P.A., Sznitman, R., and Pertz, O. (2021). CODEX, a neural network approach to explore signaling dynamics landscapes. Mol. Syst. Biol. 17, e10026.

Jaqaman, K., Loerke, D., Mettlen, M., Kuwata, H., Grinstein, S., Schmid, S.L., and Danuser, G. (2008). Robust single-particle tracking in live-cell time-lapse sequences. Nat. Methods 5 , 695-702.

Jensen, L.J., Kuhn, M., Stark, M., Chaffron, S., Creevey, C., Muller, J., Doerks, T., Julien, P., Roth, A., Simonovic, M., et al. (2009). STRING 8--a global view on proteins and their functional interactions in 630 organisms. Nucleic Acids Res. 37, D412-6.

Johnson, H.E., Goyal, Y., Pannucci, N.L., Schüpbach, T., Shvartsman, S.Y., and Toettcher, J.E. (2017). The spatiotemporal limits of developmental erk signaling. Dev. Cell 40, 185192.

Junttila, M.R., Li, S.-P., and Westermarck, J. (2008). Phosphatase-mediated crosstalk between MAPK signaling pathways in the regulation of cell survival. FASEB J. 22, 954-965.

Kholodenko, B.N. (2000). Negative feedback and ultrasensitivity can bring about oscillations in the mitogen-activted protein kinase cascades. Eur. J. Biochem 267, 1583-1588. 
Kholodenko, B.N., Hancock, J.F., and Kolch, W. (2010). Signalling ballet in space and time. Nat. Rev. Mol. Cell Biol. 11, 414-426.

Kim, N., Kim, J.M., Lee, M., Kim, C.Y., Chang, K.-Y., and Heo, W.D. (2014). Spatiotemporal control of fibroblast growth factor receptor signals by blue light. Chem. Biol. 21, 903-912.

Kochańczyk, M., Kocieniewski, P., Kozłowska, E., Jaruszewicz-Błońska, J., Sparta, B., Pargett, M., Albeck, J.G., Hlavacek, W.S., and Lipniacki, T. (2017). Relaxation oscillations and hierarchy of feedbacks in MAPK signaling. Sci. Rep. 7, 38244.

Koseska, A., and Bastiaens, P.I.H. (2020). Processing temporal growth factor patterns by an epidermal growth factor receptor network dynamically established in space. Annu. Rev. Cell Dev. Biol. 36, 359-383.

Kupzig, S., Walker, S.A., and Cullen, P.J. (2005). The frequencies of calcium oscillations are optimized for efficient calcium-mediated activation of Ras and the ERK/MAPK cascade. Proc Natl Acad Sci USA 102, 7577-7582.

Lake, D., Corrêa, S.A.L., and Müller, J. (2016). Negative feedback regulation of the ERK1/2 MAPK pathway. Cell. Mol. Life Sci. 73, 4397-4413.

Lam, A.J., St-Pierre, F., Gong, Y., Marshall, J.D., Cranfill, P.J., Baird, M.A., McKeown, M.R., Wiedenmann, J., Davidson, M.W., Schnitzer, M.J., et al. (2012). Improving FRET dynamic range with bright green and red fluorescent proteins. Nat. Methods 9, 1005-1012.

Langlois, W.J., Sasaoka, T., Saltiel, A.R., and Olefsky, J.M. (1995). Negative feedback regulation and desensitization of insulin- and epidermal growth factor-stimulated p21ras activation. J. Biol. Chem. 270, 25320-25323.

Lavoie, H., Gagnon, J., and Therrien, M. (2020). ERK signalling: a master regulator of cell behaviour, life and fate. Nat. Rev. Mol. Cell Biol. 21, 607-632.

McQuin, C., Goodman, A., Chernyshev, V., Kamentsky, L., Cimini, B.A., Karhohs, K.W., Doan, M., Ding, L., Rafelski, S.M., Thirstrup, D., et al. (2018). CellProfiler 3.0: Nextgeneration image processing for biology. PLoS Biol. 16, e2005970.

Mikelson, J., and Khammash, M. (2020). Likelihood-free nested sampling for parameter inference of biochemical reaction networks. PLoS Comput. Biol. 16, e1008264.

Nakakuki, T., Birtwistle, M.R., Saeki, Y., Yumoto, N., Ide, K., Nagashima, T., Brusch, L., Ogunnaike, B.A., Okada-Hatakeyama, M., and Kholodenko, B.N. (2010). Ligand-specific cFos expression emerges from the spatiotemporal control of ErbB network dynamics. Cell $141,884-896$.

Ng, P.K.-S., Li, J., Jeong, K.J., Shao, S., Chen, H., Tsang, Y.H., Sengupta, S., Wang, Z., Bhavana, V.H., Tran, R., et al. (2018). Systematic functional annotation of somatic mutations in cancer. Cancer Cell 33, 450-462.e10.

Ornitz, D.M., and Itoh, N. (2015). The Fibroblast Growth Factor signaling pathway. Wiley Interdiscip. Rev. Dev. Biol. 4, 215-266.

Orton, R.J., Sturm, O.E., Vyshemirsky, V., Calder, M., Gilbert, D.R., and Kolch, W. (2005). Computational modelling of the receptor-tyrosine-kinase-activated MAPK pathway. Biochem. J. 392, 249-261.

Patterson, K.I., Brummer, T., O’Brien, P.M., and Daly, R.J. (2009). Dual-specificity 
phosphatases: critical regulators with diverse cellular targets. Biochem. J. 418, 475-489.

Purvis, J.E., and Lahav, G. (2013). Encoding and decoding cellular information through signaling dynamics. Cell 152, 945-956.

Rauen, K.A. (2013). The RASopathies. Annu. Rev. Genomics Hum. Genet. 14, 355-369.

Regot, S., Hughey, J.J., Bajar, B.T., Carrasco, S., and Covert, M.W. (2014). High-sensitivity measurements of multiple kinase activities in live single cells. Cell 157, 1724-1734.

Ryu, H., Chung, M., Dobrzyński, M., Fey, D., Blum, Y., Lee, S.S., Peter, M., Kholodenko, B.N., Jeon, N.L., and Pertz, O. (2015). Frequency modulation of ERK activation dynamics rewires cell fate. Mol. Syst. Biol. 11, 838.

Saha, M., Carriere, A., Cheerathodi, M., Zhang, X., Lavoie, G., Rush, J., Roux, P.P., and Ballif, B.A. (2012). RSK phosphorylates SOS1 creating 14-3-3-docking sites and negatively regulating MAPK activation. Biochem. J. 447, 159-166.

Sakaue-Sawano, A., Kurokawa, H., Morimura, T., Hanyu, A., Hama, H., Osawa, H., Kashiwagi, S., Fukami, K., Miyata, T., Miyoshi, H., et al. (2008). Visualizing spatiotemporal dynamics of multicellular cell-cycle progression. Cell 132, 487-498.

Samatar, A.A., and Poulikakos, P.I. (2014). Targeting RAS-ERK signalling in cancer: promises and challenges. Nat. Rev. Drug Discov. 13, 928-942.

Santos, S.D.M., Verveer, P.J., and Bastiaens, P.I.H. (2007). Growth factor-induced MAPK network topology shapes Erk response determining PC-12 cell fate. Nat. Cell Biol. 9, 324330.

Schlessinger, J. (2000). Cell signaling by receptor tyrosine kinases. Cell 103, 211-225.

Schmick, M., Kraemer, A., and Bastiaens, P.I.H. (2015). Ras moves to stay in place. Trends Cell Biol. 25, 190-197.

Schwanhäusser, B., Busse, D., Li, N., Dittmar, G., Schuchhardt, J., Wolf, J., Chen, W., and Selbach, M. (2011). Global quantification of mammalian gene expression control. Nature 473, 337-342.

Shankaran, H., Ippolito, D.L., Chrisler, W.B., Resat, H., Bollinger, N., Opresko, L.K., and Wiley, H.S. (2009). Rapid and sustained nuclear-cytoplasmic ERK oscillations induced by epidermal growth factor. Mol. Syst. Biol. 5, 332.

Shcherbakova, D.M., Baloban, M., Emelyanov, A.V., Brenowitz, M., Guo, P., and Verkhusha, V.V. (2016). Bright monomeric near-infrared fluorescent proteins as tags and biosensors for multiscale imaging. Nat. Commun. 7, 12405.

Smith, J.A., Poteet-Smith, C.E., Xu, Y., Errington, T.M., Hecht, S.M., and Lannigan, D.A. (2005). Identification of the first specific inhibitor of p90 ribosomal S6 kinase (RSK) reveals an unexpected role for RSK in cancer cell proliferation. Cancer Res. 65, 1027-1034.

Sturm, O.E., Orton, R., Grindlay, J., Birtwistle, M., Vyshemirsky, V., Gilbert, D., Calder, M., Pitt, A., Kholodenko, B., and Kolch, W. (2010). The mammalian MAPK/ERK pathway exhibits properties of a negative feedback amplifier. Sci. Signal. 3, ra90.

Wei, W., Jiao, Y., Postlethwaite, A., Stuart, J.M., Wang, Y., Sun, D., and Gu, W. (2013). Dual-specificity phosphatases 2: surprising positive effect at the molecular level and a potential biomarker of diseases. Genes Immun. 14, 1-6. 
Yarden, Y., and Pines, G. (2012). The ERBB network: at last, cancer therapy meets systems biology. Nat. Rev. Cancer 12, 553-563.

York, R.D., Yao, H., Dillon, T., Ellig, C.L., Eckert, S.P., McCleskey, E.W., and Stork, P.J. (1998). Rap1 mediates sustained MAP kinase activation induced by nerve growth factor. Nature 392, 622-626.

Yusa, K., Zhou, L., Li, M.A., Bradley, A., and Craig, N.L. (2011). A hyperactive piggyBac transposase for mammalian applications. Proc Natl Acad Sci USA 108, 1531-1536. 